\title{
TetrazineBox: A Structurally Transformative ToolBox
}

Qing-Hui Guo, ${ }^{\dagger}$ Jiawang Zhou, ${ }^{\dagger \dagger}$ Haochuan Mao, ${ }^{\dagger \dagger}$ Yunyan Qiu, ${ }^{\dagger}$ Minh T. Nguyen, ${ }^{\dagger}$ Yuanning Feng, ${ }^{\dagger}$ Jiaqi Liang, ${ }^{\dagger}$ Dengke Shen, ${ }^{\dagger}$ Penghao Li,${ }^{\dagger}$ Zhichang Liu, ${ }^{\ddagger}$

Michael R. Wasielewski, ${ }^{\dagger, *}$ and J. Fraser Stoddart*,+, \#

'Department of Chemistry, Northwestern University, 2145 Sheridan Road, Evanston, Illinois 60208, USA

Institute for Sustainability and Energy at Northwestern, Northwestern University, 2145 Sheridan Road, Evanston, Illinois 60208, USA

${ }^{\sharp}$ School of Science, Westlake University, 18 Shilongshan Road, Hangzhou 310024, China

§Institute for Molecular Design and Synthesis, Tianjin University, 92 Weijin Road, Nankai District, Tianjin 300072, China

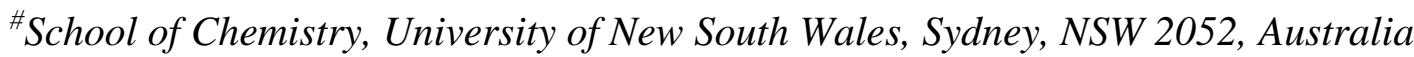
*E-mail: stoddart@northwestern.edu

\section{Supporting Information}

\section{Table of Contents}

Section A. General Information ..........................................................................2

Section B. Synthetic Protocols .............................................................................................3

Section C. X-Ray Crystallographic Characterization .................................................8

Section D. Redox Chemistry and UV-Vis-NIR Spectra.......................................11

Section E. Regulatable Binding Affinities.....................................................15

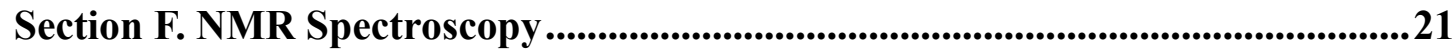

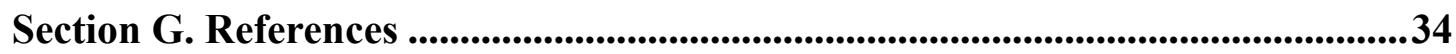




\section{Section A. General Information}

All commercially available reagents were used as received. Anhydrous $\mathrm{MeCN}$ and $\mathrm{CH}_{2} \mathrm{Cl}_{2}$ were prepared by solvent drying system. 3,6-Di(4-pyridinyl)-1,2,4,5-tetrazine (1) was synthesized according to literature ${ }^{1}$. Bis(cyclopentadienyl)cobalt(II) was purchased from Sigma-Aldrich. UV-Vis-NIR absorption spectra were collected on a Shimadzu UV-3600 spectrophotometer. Nuclear magnetic resonance (NMR) spectra were recorded on Bruker Avance 500 spectrometers, with working frequencies of 500 $\mathrm{MHz}$ for ${ }^{1} \mathrm{H}$ and $125 \mathrm{MHz}$ for ${ }^{13} \mathrm{C}$ nuclei, respectively. Chemical shifts were reported in ppm relative to the signals corresponding to the residual non-deuterated solvents $\left(\mathrm{DMSO}-d_{6}: \delta_{\mathrm{H}}=2.50\right.$ and $\delta_{\mathrm{C}}=39.5 \mathrm{ppm} ; \mathrm{CD}_{3} \mathrm{CN}: \delta_{\mathrm{H}}=1.94$ and $\delta_{\mathrm{C}}=118.3 \mathrm{ppm}$ ). Abbreviations are used in the description of NMR data as follows: chemical shift $(\delta$, ppm), multiplicity $(\mathrm{s}=$ singlet, $\mathrm{d}=$ doublet $)$, coupling constant $(J, \mathrm{~Hz})$. EPR Spectra were recorded using a Bruker Elexsys E580 X-band EPR spectrometer. EPR Samples were prepared by titration with cobaltocene and the solution was transferred into 1.8 mm o.d. quartz tubes and sealed with a clear ridged UV doming epoxy in an Ar glovebox. High-resolution mass spectra (ESI-HRMS) were measured on a Finnigan LCQ iontrap mass spectrometer. Single-crystal X-ray diffraction (SCXRD) data were collected on a Bruker APEX-II CCD diffractometer. 


\section{Section B. Synthetic Protocols}

\section{Synthesis of $2 \cdot 2 P_{6}$}

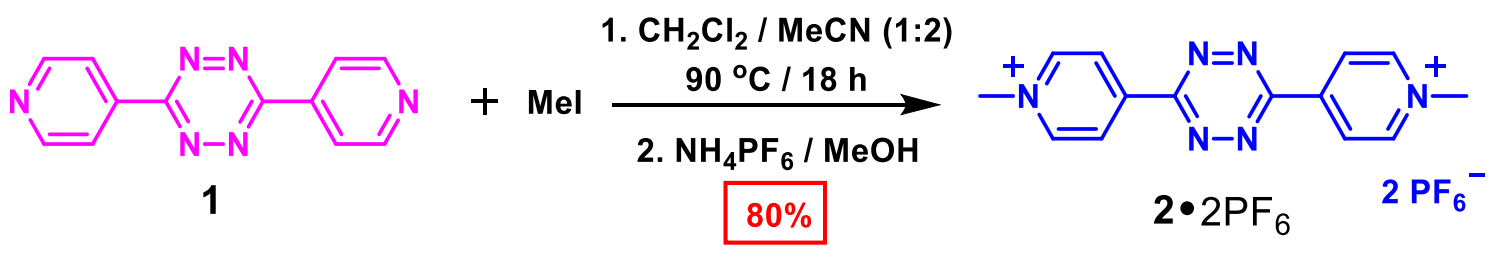

Scheme S1. Synthesis of $2 \cdot 2 \mathrm{PF}_{6}$

2•2PF6: A solution of 3,6-di(4-pyridinyl)-1,2,4,5-tetrazine 1 (118 mg, $0.5 \mathrm{mmol})$ and methyl iodide $(710 \mathrm{mg}, 5 \mathrm{mmol})$ in the mixture of dry $\mathrm{CH}_{2} \mathrm{Cl}_{2}(20 \mathrm{~mL})$ and anhydrous $\mathrm{MeCN}(40 \mathrm{~mL})$ was heated to $90{ }^{\circ} \mathrm{C}$ for $18 \mathrm{~h}$. The resulting red precipitate was collected by filtration, and washed with $\mathrm{CH}_{2} \mathrm{Cl}_{2}(3 \times 10 \mathrm{~mL})$. The precipitate was then dissoved in anhydrous $\mathrm{MeOH}(200 \mathrm{~mL})$ and the solution was filtered. A solution of $\mathrm{NH}_{4} \mathrm{PF}_{6}(2$ $\mathrm{g})$ in anhydrous $\mathrm{MeOH}(20 \mathrm{~mL})$ was added in the filtrate. The resulting red precipitate was collected by filtration, washed with $\mathrm{CH}_{2} \mathrm{Cl}_{2}(3 \times 20 \mathrm{~mL})$ and dried to give the product 2 $2 \mathrm{PF}_{6}(222 \mathrm{mg}, 0.4 \mathrm{mmol})$ in $80 \%$ yield. ${ }^{1} \mathrm{H} \mathrm{NMR}\left(500 \mathrm{MHz}, \mathrm{CD}_{3} \mathrm{CN}, 298\right.$ K) $\delta_{H}=9.16(\mathrm{~d}, J=6.17 \mathrm{~Hz}, 4 \mathrm{H}), 9.00(\mathrm{~d}, J=6.17 \mathrm{~Hz}, 4 \mathrm{H}), 4.49(\mathrm{~s}, 4 \mathrm{H}) ;{ }^{13} \mathrm{C} \mathrm{NMR}$ $\left(125 \mathrm{MHz}, \mathrm{CD}_{3} \mathrm{SOCD}_{3}, 298 \mathrm{~K}\right) \delta_{C}=161.8,147.9,145.9,126.0,125.6,49.0$; ESIHRMS Calcd for $\mathrm{C}_{14} \mathrm{H}_{14} \mathrm{~F}_{6} \mathrm{~N}_{6} \mathrm{P}: m / z=411.0916[M+\mathrm{H}]^{+}$; found: $411.0917[M+\mathrm{H}]^{+}$. 


\section{Synthesis of $3 \cdot 2 P F_{6}$}

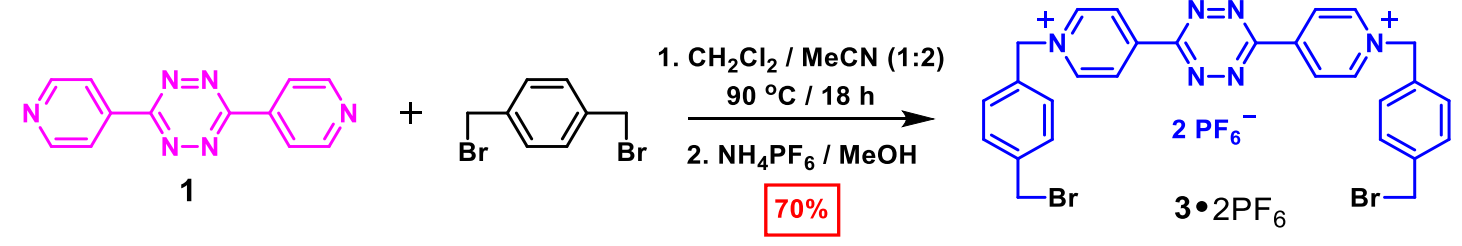

Scheme S2. Synthesis of $3 \cdot 2 \mathrm{PF}_{6}$

3•2PF6: A solution of $\alpha, \alpha^{\prime}$-dibromo-p-xylene (13.20 g, $\left.50 \mathrm{mmol}\right)$ and 3,6-di(4pyridinyl)-1,2,4,5-tetrazine 1 (1.18 g, $5 \mathrm{mmol})$ in the mixture of dry $\mathrm{CH}_{2} \mathrm{Cl}_{2}(80 \mathrm{~mL})$ and anhydrous $\mathrm{MeCN}(160 \mathrm{~mL})$ was heated to $90{ }^{\circ} \mathrm{C}$ for $18 \mathrm{~h}$. The resulting red precipitate was collected by filtration, washed with $\mathrm{CH}_{2} \mathrm{Cl}_{2}(3 \times 20 \mathrm{~mL})$. The precipitate was then dissoved in anhydrous $\mathrm{MeOH}(400 \mathrm{~mL})$ and the solution was filtered. A solution of $\mathrm{NH}_{4} \mathrm{PF}_{6}(8 \mathrm{~g})$ in anhydrous $\mathrm{MeOH}(50 \mathrm{~mL})$ was added in the filtrate. The resulting red precipitate was collected by filtration, washed with $\mathrm{CH}_{2} \mathrm{Cl}_{2}(3 \times 20 \mathrm{~mL})$ and dried to give the product $3 \cdot 2 \mathrm{PF}_{6}(3.13 \mathrm{~g}, 3.5 \mathrm{mmol})$ in $70 \%$ yield. ${ }^{1} \mathrm{H}$ NMR (500 $\left.\mathrm{MHz}, \mathrm{CD}_{3} \mathrm{CN}, 298 \mathrm{~K}\right) \delta_{H}=9.17(\mathrm{~d}, J=6.46 \mathrm{~Hz}, 4 \mathrm{H}), 9.10(\mathrm{~d}, J=6.46 \mathrm{~Hz}, 4 \mathrm{H}), 7.60$ $(\mathrm{d}, J=8.02 \mathrm{~Hz}, 4 \mathrm{H}), 7.55(\mathrm{~d}, J=8.02 \mathrm{~Hz}, 4 \mathrm{H}), 5.91(\mathrm{~s}, 4 \mathrm{H}), 4.65(\mathrm{~s}, 4 \mathrm{H}) ;{ }^{13} \mathrm{C} \mathrm{NMR}$ $\left(125 \mathrm{MHz}, \mathrm{CD}_{3} \mathrm{CN}, 298 \mathrm{~K}\right) \delta_{C}=162.3,147.6,146.8,141.1,133.0,130.8,130.5,127.5$, 65.1, 33.1; ESI-HRMS Calcd for $\mathrm{C}_{28} \mathrm{H}_{24} \mathrm{Br}_{2} \mathrm{~N}_{6} \mathrm{~F}_{12} \mathrm{P}_{2}: m / z=749.0045\left[M-\mathrm{PF}_{6}\right]^{+}$; found: 749.0081 $\left[M-\mathrm{PF}_{6}\right]^{+}$. 


\section{Synthesis of $4 \cdot 4 \mathrm{PF}_{6}$}
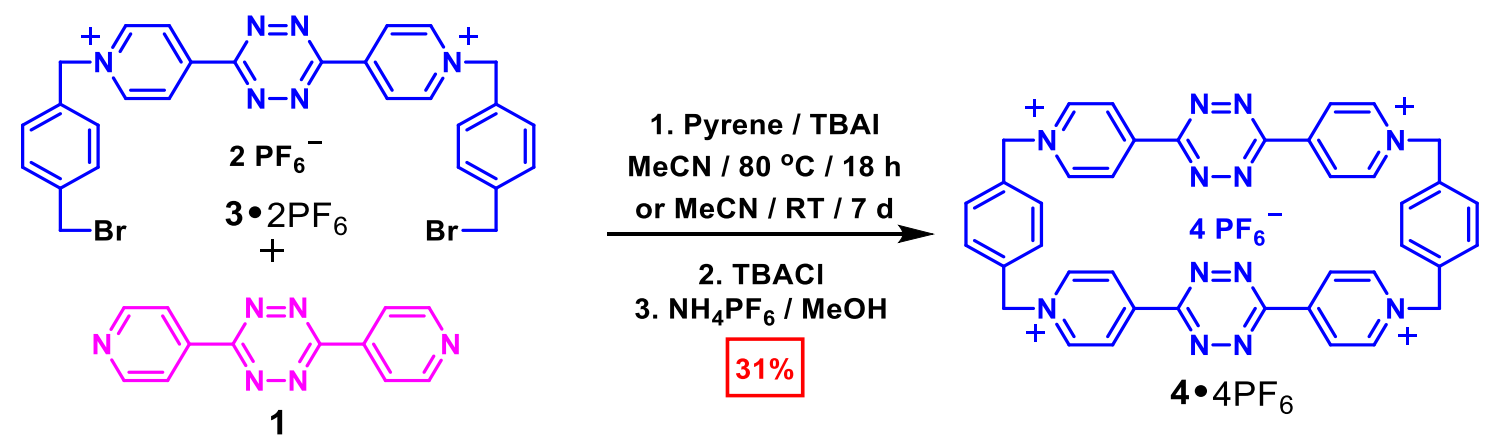

Scheme S3. Synthesis of $4 \bullet 4 \mathrm{PF}_{6}$

4•4PF6: A template pyrene $(1.51 \mathrm{~g}, 6 \mathrm{mmol})$ and a catalyst TBAI $(111 \mathrm{mg}, 0.3 \mathrm{mmol})$ were added to a solution of $\mathbf{1}(894 \mathrm{~g}, 1 \mathrm{mmol})$ and $\mathbf{3} \cdot 2 \mathrm{PF}_{6}$ in anhydrous $\mathrm{MeCN}$ (500 $\mathrm{mL}$ ). The mixture was heated to $80{ }^{\circ} \mathrm{C}$ for $18 \mathrm{~h}$ or stirred at room temperature for 7 days. The resulting red precipitate was collected by filtration, washed with $\mathrm{CH}_{2} \mathrm{Cl}_{2}(3 \times 20$ $\mathrm{mL})$. The precipitate was then dissoved in anhydrous $\mathrm{MeOH}(800 \mathrm{~mL})$ and the solution was filtered. A solution of $\mathrm{NH}_{4} \mathrm{PF}_{6}(2.5 \mathrm{~g})$ in anhydrous $\mathrm{MeOH}(50 \mathrm{~mL})$ was added to the filtrate. The resulting red precipitate was collected by filtration, washed with $\mathrm{CH}_{2} \mathrm{Cl}_{2}(3 \times 20 \mathrm{~mL})$ and dried. The analytically pure product $4 \cdot 4 \mathrm{PF}_{6}(391 \mathrm{mg}, 0.31$ mmol), which was obtained in $31 \%$ yield, was isolated as a red powder by highperformance reverse-phase preparative C18 column chromatography (anhydrous $\mathrm{MeCN}$ and $5 \% \mathrm{CH}_{2} \mathrm{Cl}_{2}$ as eluent). ${ }^{1} \mathrm{H}$ NMR $\left(500 \mathrm{MHz}, \mathrm{CD}_{3} \mathrm{CN}, 298 \mathrm{~K}\right) \delta_{H}=8.93(\mathrm{~d}, J$ $=7.00 \mathrm{~Hz}, 8 \mathrm{H}), 8.62(\mathrm{~d}, J=7.00 \mathrm{~Hz}, 8 \mathrm{H}), 7.67(\mathrm{~s}, 8 \mathrm{H}), 7.67(\mathrm{~s}, 8 \mathrm{H}) ;{ }^{13} \mathrm{C} \mathrm{NMR}(125$ $\left.\mathrm{MHz}, \mathrm{CD}_{3} \mathrm{CN}, 298 \mathrm{~K}\right) \delta_{C}=154.8,151.5,145.4,136.5,130.9,127.8,126.8,64.9,22.8$; ESI-HRMS Calcd for $\mathrm{C}_{40} \mathrm{H}_{32} \mathrm{~F}_{24} \mathrm{~N}_{12} \mathrm{P}_{4}: m / z=1115.1793\left[M-\mathrm{PF}_{6}\right]^{+}, 485.1073[M-$ $\left.2 \mathrm{PF}_{6}\right]^{2+}$; found: $1115.1782\left[M-\mathrm{PF}_{6}\right]^{+}, 485.1077\left[M-2 \mathrm{PF}_{6}\right]^{2+}$. 


\section{Synthesis of $5 \bullet 4 P_{6}$}
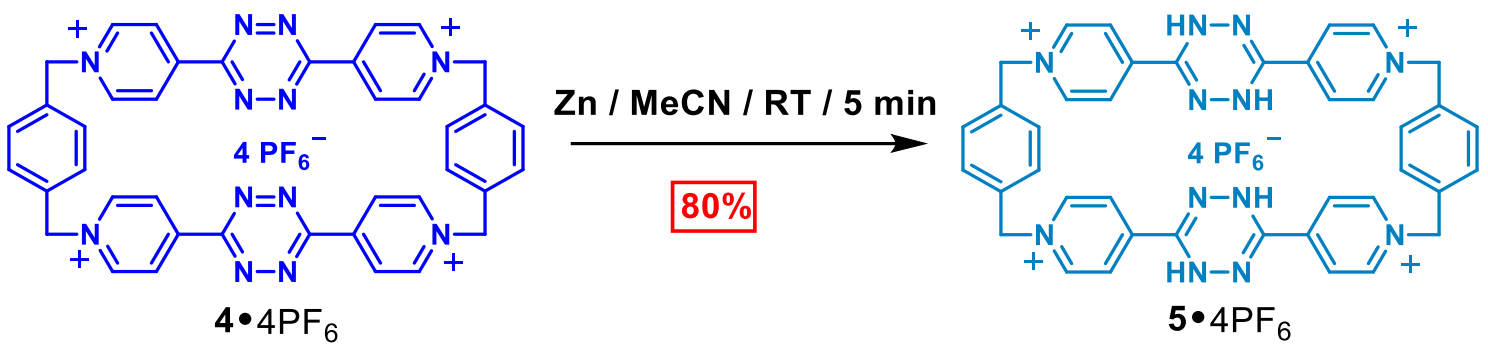

Scheme S4. Synthesis of $\mathbf{5} \bullet 4 \mathrm{PF}_{6}$

5•4PF6: Excess of activated $\mathrm{Zn}$ powder was added to a solution of 4•4PF $6(10 \mathrm{mg}, 0.008$ mmol) in anhydrous $\mathrm{MeCN}(5 \mathrm{~mL})$ in a $\mathrm{N}_{2}$ glovebox. The mixture was stirred at room temperature for $5 \mathrm{~min}$. The $\mathrm{Zn}$ powder was filtrered off and the resulting clear filtrate was allowed to stand in a 1-ml vial in a $\mathrm{N}_{2}$ glovebox. On slow vapor diffusion of $i \operatorname{Pr}_{2} \mathrm{O}$ into the $\mathrm{MeCN}$ solution during one day, the pure product $5 \bullet 4 \mathrm{PF}_{6}(8 \mathrm{mg}, 0.0064 \mathrm{mmol})$ was afforded as red crystals in $80 \%$ yield. ${ }^{1} \mathrm{H}$ NMR $\left(500 \mathrm{MHz}, \mathrm{CD}_{3} \mathrm{CN}, 298 \mathrm{~K}\right) \delta_{H}=$ $8.79(\mathrm{~d}, J=7.02 \mathrm{~Hz}, 4 \mathrm{H}), 8.55(\mathrm{~s}, 8 \mathrm{H}), 8.15(\mathrm{~d}, J=7.02 \mathrm{~Hz}, 8 \mathrm{H}), 7.56(\mathrm{~s}, 8 \mathrm{H}), 5.73(\mathrm{~s}$, $8 \mathrm{H}) ;{ }^{13} \mathrm{C}$ NMR $\left(125 \mathrm{MHz}, \mathrm{CD}_{3} \mathrm{CN}, 298 \mathrm{~K}\right) \delta_{C}=145.9,145.4,143.2,135.7,130.8$, $125.5,64.6$

\section{Synthesis of $6 \bullet 4 \mathrm{PF}_{6}$}
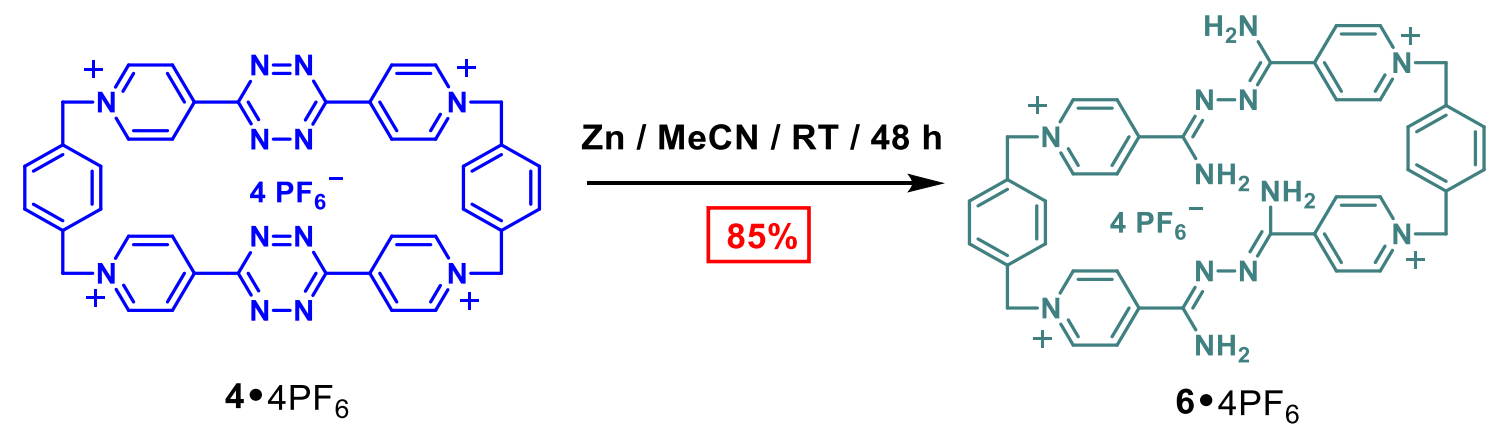

Scheme S5. Synthesis of $6 \bullet 4 \mathrm{PF}_{6}$

6•4PF6: Excess of activated $\mathrm{Zn}$ powder was added to a solution of $\mathbf{4} \cdot 4 \mathrm{PF}_{6}(100 \mathrm{mg}$, $0.08 \mathrm{mmol})$ in anhydrous $\mathrm{MeCN}(25 \mathrm{~mL})$ in a $\mathrm{N}_{2}$ glovebox. The mixture was stirred at room temperature for $48 \mathrm{~h}$. The $\mathrm{Zn}$ powder was filtrered off, the resulting clear filtrate 
was subjected to $\mathrm{SiO}_{2}$ column chromatography $\left(0.25 \% \mathrm{NH}_{4} \mathrm{PF}_{6}\right.$ in $\mathrm{MeCN}$ as eluent). The pure product $6 \cdot 4 \mathrm{PF}_{6}(86 \mathrm{mg}, 0.068 \mathrm{mmol})$ was afforded as a red powder in $85 \%$ yield. ${ }^{1} \mathrm{H}$ NMR $\left(500 \mathrm{MHz}, \mathrm{CD}_{3} \mathrm{CN}, 298 \mathrm{~K}\right) \delta_{H}=8.75(\mathrm{~d}, J=6.48 \mathrm{~Hz}, 8 \mathrm{H}), 8.33(\mathrm{~d}, J=$ $6.48 \mathrm{~Hz}, 8 \mathrm{H}), 7.59(\mathrm{~s}, 8 \mathrm{H}), 6.34(\mathrm{~s}, 8 \mathrm{H}), 5.66(\mathrm{~s}, 8 \mathrm{H}) ;{ }^{13} \mathrm{C} \mathrm{NMR}\left(125 \mathrm{MHz}, \mathrm{CD}_{3} \mathrm{CN}\right.$, $298 \mathrm{~K}) \delta_{C}=152.0,149.9,144.3,136.6,130.7,125.6,64.7$; ESI-HRMS Calcd for $\mathrm{C}_{40} \mathrm{H}_{32} \mathrm{~F}_{24} \mathrm{~N}_{12} \mathrm{P}_{4}: m / z=1115.1793\left[M-\mathrm{PF}_{6}\right]^{+}, 485.1073\left[M-2 \mathrm{PF}_{6}\right]^{2+}$; found: 1115.1782 $\left[M-\mathrm{PF}_{6}\right]^{+}, 485.1077\left[M-2 \mathrm{PF}_{6}\right]^{2+}$.

\section{Synthesis of $7 \cdot 4 \mathrm{PF}_{6}$}
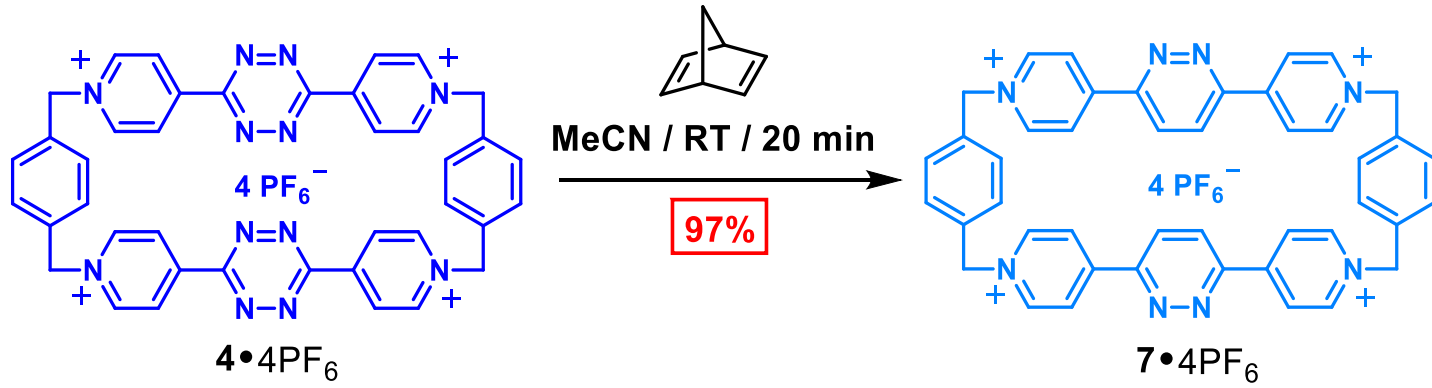

Scheme S6. Synthesis of 7•4PF 6

7•4PF $\mathbf{6}$ : Excess of norbornadiene $(147 \mathrm{mg}, 1.6 \mathrm{mmol})$ was added to a solution of $4 \cdot 4 \mathrm{PF}_{6}$ (100 mg, $0.08 \mathrm{mmol})$ in anhydrous $\mathrm{MeCN}(25 \mathrm{~mL})$. The mixture was stirred at room temperature for 20 min while $\mathrm{N}_{2}$ was released. The mixture was subjected to $\mathrm{SiO}_{2}$ column chromatography $\left(0.25 \% \mathrm{NH}_{4} \mathrm{PF}_{6}\right.$ in $\mathrm{MeCN}$ as eluent $)$. The pure product $7 \bullet 4 \mathrm{PF}_{6}$ (86 mg, $0.068 \mathrm{mmol}$ ) was afforded as a yellow powder in 97\% yield. ${ }^{1} \mathrm{H}$ NMR (500 $\left.\mathrm{MHz}, \mathrm{CD}_{3} \mathrm{CN}, 298 \mathrm{~K}\right) \delta_{H}=8.90(\mathrm{~d}, J=6.92 \mathrm{~Hz}, 4 \mathrm{H}), 8.60(\mathrm{~d}, J=6.92 \mathrm{~Hz}, 8 \mathrm{H}), 8.44$ $(\mathrm{s}, 4 \mathrm{H}), 7.63(\mathrm{~s}, 8 \mathrm{H}), 5.77(\mathrm{~s}, 8 \mathrm{H}) ;{ }^{13} \mathrm{C} \mathrm{NMR}\left(125 \mathrm{MHz}, \mathrm{CD}_{3} \mathrm{CN}, 298 \mathrm{~K}\right) \delta_{C}=154.8$, 151.5, 145.4, 136.5, 130.9, 127.8, 126.8, 64.9.ESI-HRMS Calcd for $\mathrm{C}_{44} \mathrm{H}_{36} \mathrm{~F}_{24} \mathrm{~N}_{8} \mathrm{P}_{4}$ : $m / z=1111.1983\left[M-\mathrm{PF}_{6}\right]^{+} ;$found: $1111.1994\left[M-\mathrm{PF}_{6}\right]^{+}$. 


\section{Section C. X-Ray Crystallographic Characterization}

All the single crystals were obtained by slow vapor diffusion of $i \operatorname{Pr}_{2} \mathrm{O}$ into solutions of 2•2PF $6,4 \cdot 4 \mathrm{PF}_{6}, \mathbf{5} \cdot 4 \mathrm{PF}_{6}, \mathbf{6} \cdot 4 \mathrm{PF}_{6}$, and $7 \cdot 4 \mathrm{PF}_{6}$ in $\mathrm{MeCN}$ after two days, respectively. Single crystals of host-guest complexes (pyrene $\subset$ TzBox $\bullet 4 \mathrm{PF}_{6}$, perylene $\subset$ TzBox$\bullet 4 \mathrm{PF}_{6}$, pyrene $\subset \mathrm{DzBox} \cdot 4 \mathrm{PF}_{6}$, and perylene $\subset$ DzBox$\bullet 4 \mathrm{PF}_{6}$ ) were obtained by slow vapor diffusion of $i \operatorname{Pr}_{2} \mathrm{O}$ into $\mathrm{MeCN}$ solutions containing 1:1 ratios of hosts to guests at room temperature. Single crystals suitable for X-ray crystallography were selected and mounted in an inert oil and transferred to the cold $\mathrm{N}_{2}$ gas stream of a Bruker Kappa APEX CCD area detector diffractometer. The crystals were kept at 100 $\mathrm{K}$ during the data collection. Using Olex $2^{2}$, structures were resolved with the $\mathrm{XT}^{3}$ structure solution program employing Dual Space and refined with the $\mathrm{XL}^{4}$ refinement package using least squares minimization. All crystallographic data for the structures reported here have been deposited on to the Cambridge Crystallographic Data Centre (CCDC) and can be downloaded free of charge via www.ccdc.cam.ac.uk/data_request/cif. The crystallographic information, structural parameters for $\mathbf{2} \cdot 2 \mathrm{PF}_{6}, \quad \mathbf{4} \bullet 4 \mathrm{PF}_{6}, \mathbf{5} \cdot 4 \mathrm{PF}_{6}, \mathbf{6} \cdot 4 \mathrm{PF}_{6}, \mathbf{7} \cdot 4 \mathrm{PF}_{6}, \quad$ pyrene $\subset$ TzBox$\bullet 4 \mathrm{PF}_{6}$, perylene $\subset$ TzBox $\bullet 4 \mathrm{PF}_{6}$, pyrene $\subset \mathrm{DzBox} \cdot 4 \mathrm{PF}_{6}$, and perylene $\subset \mathrm{DzBox} \cdot 4 \mathrm{PF}_{6}$ are as follows.

2•2PF $\mathrm{P}_{6}$ Crystal Parameters. $\left[\mathrm{C}_{14} \mathrm{H}_{14} \mathrm{~F}_{12} \mathrm{~N}_{6} \mathrm{P}_{2}\right]$. Triclinic, space group $P-1, a=7.8767(6)$, $b=9.0304(8), c=14.5443(12) \AA, \alpha=75.627(4), \beta=82.233(4), \gamma=82.011(4)^{\circ}, V=$ 986.95(14) $\AA^{3}, Z=2, T=100(2) \mathrm{K}, \rho_{\text {calc }}=1.872 \mathrm{~g} \mathrm{~cm}^{-3}$. The final $R_{1}$ was 0.0277 and $w R_{2}$ was 0.0714 (all data). CCDC number 1972857.

4•4PF 6 Crystal Parameters. $\left[\mathrm{C}_{54} \mathrm{H}_{53} \mathrm{~F}_{24} \mathrm{~N}_{19} \mathrm{P}_{4}\right]$. Monoclinic, space group $P 12_{1} / c 1, a=$ 17.4620(7), $b=20.4049(7), c=19.1126(7) \AA, \alpha=90, \beta=90.954(2), \gamma=90^{\circ}, V=$ 6809.1(5) $\AA^{3}, Z=4, T=100(2) \mathrm{K}, \rho_{\text {calc }}=1.510 \mathrm{~g} \mathrm{~cm}^{-3}$. The final $R_{1}$ was 0.0628 and $w R_{2}$ was 0.1770 (all data). CCDC number 1972858.

5•4PF 6 Crystal Parameters. $\left[\mathrm{C}_{26} \mathrm{H}_{27} \mathrm{~F}_{12} \mathrm{~N}_{9} \mathrm{P}_{2}\right]$. Triclinic, space group $P-1, a=10.3447(4)$, 
$b=11.4860(4), c=17.3882(6) \AA, \alpha=104.290(2), \beta=94.193(2), \gamma=112.848(2)^{\circ}, V=$ 1811.71(12) $\AA^{3}, Z=2, T=100(2) \mathrm{K}, \rho_{\text {calc }}=1.385 \mathrm{~g} \mathrm{~cm}^{-3}$. The final $R_{1}$ was 0.0516 and $w R_{2}$ was 0.1422 (all data). CCDC number 1972862.

6•4PF 6 Crystal Parameters. $\left[\mathrm{C}_{40} \mathrm{H}_{40} \mathrm{~F}_{18} \mathrm{~N}_{12} \mathrm{P}_{3}\right]$. Monoclinic, space group $C 12 / c 1, a=$ 16.1323(5), $b=11.4829(4), c=30.2137(9) \AA, \alpha=90, \beta=101.490(2), \gamma=90^{\circ}, V=$ 5484.8(3) $\AA^{3}, Z=4, T=100(2) \mathrm{K}, \rho_{\text {calc }}=1.361 \mathrm{~cm}^{-3}$. The final $R_{1}$ was 0.0405 and $w R_{2}$ was 0.1091 (all data). CCDC number 1972863.

7•4PF 6 Crystal Parameters. [ $\left.\mathrm{C}_{46} \mathrm{H}_{39} \mathrm{~F}_{24} \mathrm{~N}_{9} \mathrm{P}_{4}\right]$. Triclinic, space group $P-1, a=13.3898(4)$, $b=14.5809(4), c=15.0625(4) \AA, \alpha=106.7410(10), \beta=99.5330(10), \gamma=110.0920(10)^{\circ}$, $V=2527.68(12) \AA^{3}, Z=2, T=100(2) \mathrm{K}, \rho_{\text {calc }}=1.705 \mathrm{~g} \mathrm{~cm}^{-3}$. The final $R_{1}$ was 0.0607 and $w R_{2}$ was 0.1704 (all data). CCDC number 1972859.

Pyrene $\subset$ TzBox $\bullet 4 \mathrm{PF}_{6}$ Crystal Parameters. $\left[\mathrm{C}_{64} \mathrm{H}_{54} \mathrm{~F}_{246} \mathrm{~N}_{18} \mathrm{P}_{4}\right]$. Triclinic, space group $P-1, a=10.8605(4), b=13.1591(5), c=13.4694(5) \AA, \alpha=81.307(2), \beta=87.8564(18)$, $\gamma=67.7952(17)^{\mathrm{o}}, V=1761.33(12) \AA^{3}, Z=1, T=100(2) \mathrm{K}, \rho_{\text {calc }}=1.534 \mathrm{~g} \mathrm{~cm}^{-3}$. The final $R_{1}$ was 0.0644 and $w R_{2}$ was 0.1839 (all data). CCDC number 1972860 .

Perylene $\subset$ TzBox $\bullet 4 \mathrm{PF}_{6}$ Crystal Parameters. $\left[\mathrm{C}_{68} \mathrm{H}_{56} \mathrm{~F}_{24} \mathrm{~N}_{16} \mathrm{P}_{4}\right]$. Triclinic, space group $P-1, a=10.8276(8), b=13.2652(9), c=13.6121(10) \AA, \alpha=81.996(3), \beta=89.523(3)$, $\gamma=67.586(3)^{\circ}, V=1787.7(2) \AA^{3}, Z=1, T=100(2) \mathrm{K}, \rho_{\text {calc }}=1.558 \mathrm{~g} \mathrm{~cm}^{-3}$. The final $R_{1}$ was 0.0507 and $w R_{2}$ was 0.1403 (all data). CCDC number 1972864.

Pyrene $\subset$ DzBox$\bullet 4 \mathrm{PF}_{6}$ Crystal Parameters. $\left[\mathrm{C}_{68} \mathrm{H}_{58} \mathrm{~F}_{24} \mathrm{~N}_{12} \mathrm{P}_{4}\right]$. Triclinic, space group $P-1, a=10.4268(5), b=11.1641(5), c=17.3653(8) \AA, \alpha=74.541(3), \beta=80.014(3)$, $\gamma=63.639(2)^{\mathrm{o}}, V=1742.15(15) \AA^{3}, Z=1, T=100(2) \mathrm{K}, \rho_{\text {calc }}=1.547 \mathrm{~g} \mathrm{~cm}^{-3}$. The final $R_{1}$ was 0.0609 and $w R_{2}$ was 0.1699 (all data). CCDC number 1972861.

Perylene $\subset$ DzBox $\bullet 4 \mathrm{PF}_{6}$ Crystal Parameters. $\left[\mathrm{C}_{72} \mathrm{H}_{60} \mathrm{~F}_{24} \mathrm{~N}_{12} \mathrm{P}_{4}\right]$. Triclinic, space group $P-1, a=10.4662(5), b=11.0351(5), c=17.4251(8) \AA, \alpha=74.1610(15), \beta=$ $78.6350(15), \gamma=65.1600(10)^{\circ}, V=1749.15(14) \AA^{3}, Z=1, T=100(2) \mathrm{K}, \rho_{\text {calc }}=1.5883$ $\mathrm{g} \mathrm{cm}^{-3}$. The final $R_{1}$ was 0.0454 and $w R_{2}$ was 0.1191 (all data). CCDC number 1972865 . 
a
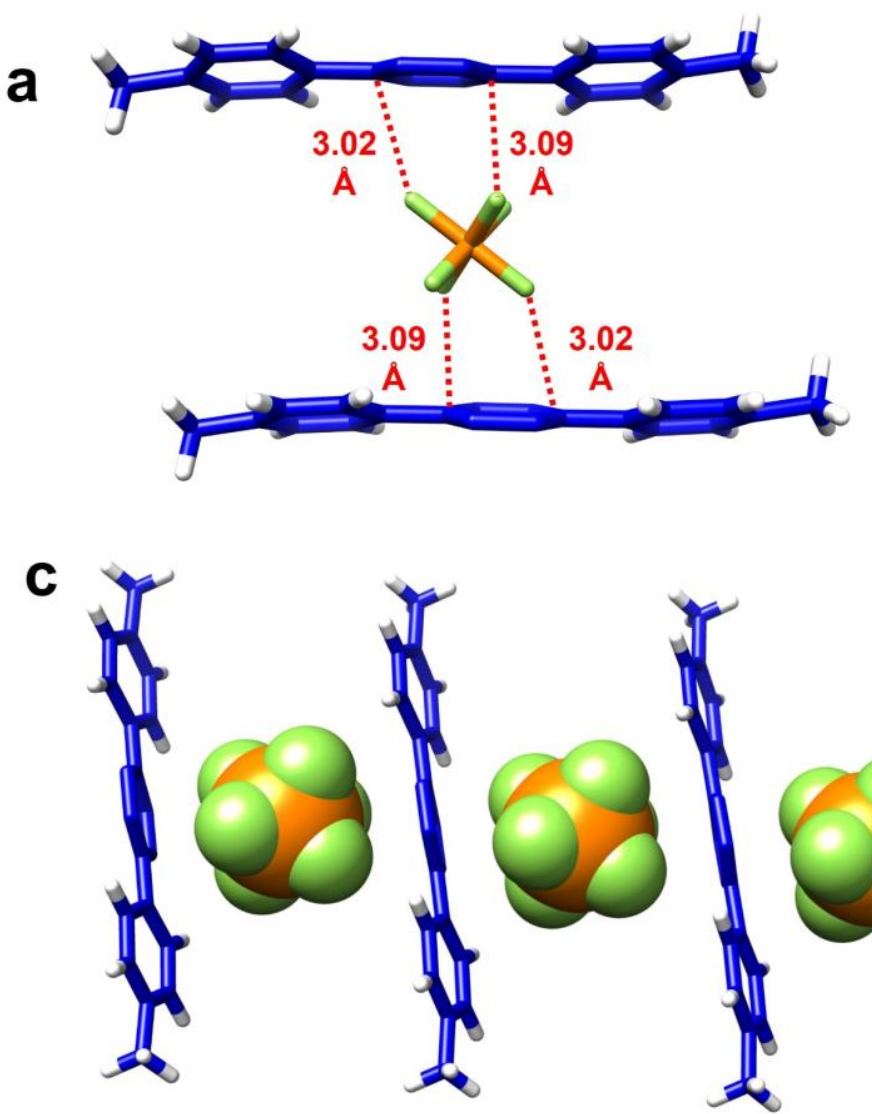
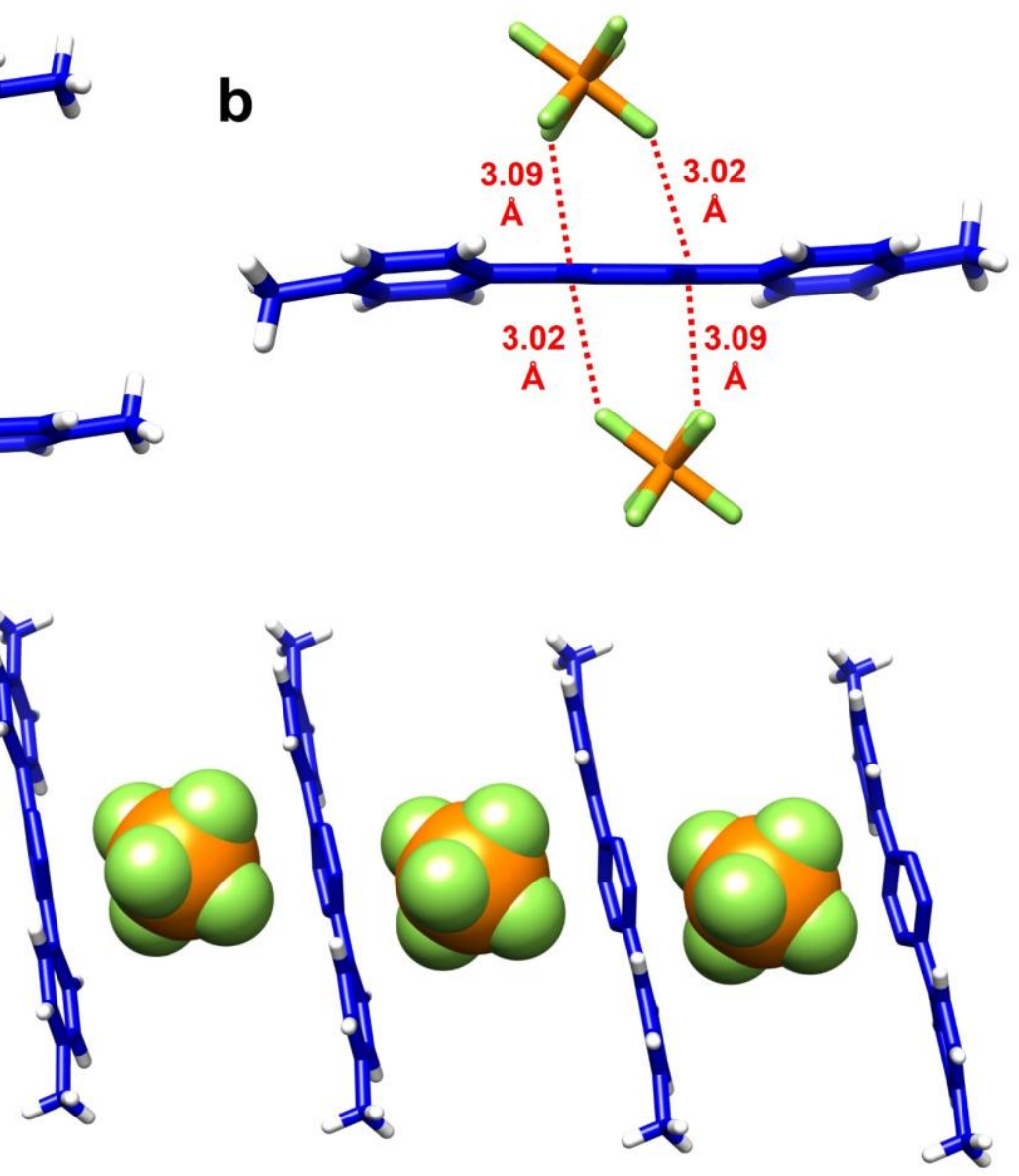

Figure S1. The multiple anion- $\pi$ interactions between $\mathrm{PF}_{6}{ }^{-}$counterions and $\pi$ electron-deficient $\mathbf{2}^{2+}$ fragments in the superstructures of $2 \cdot 2 \mathrm{PF}_{6}$. (a) One $\mathrm{PF}_{6}{ }^{-}$counterion interacts with two $2^{2+}$ fragments. (b) One $\mathbf{2}^{2+}$ fragment interacts with two $\mathrm{PF}_{6}{ }^{-}$counterions. (c) $\mathbf{2}^{2+}$ fragments and $\mathrm{PF}_{6}{ }^{-}$ counterions assemble into a $1 \mathrm{D}$ superstructure. 
Section D. Redox Chemistry and UV-Vis-NIR Spectra

a
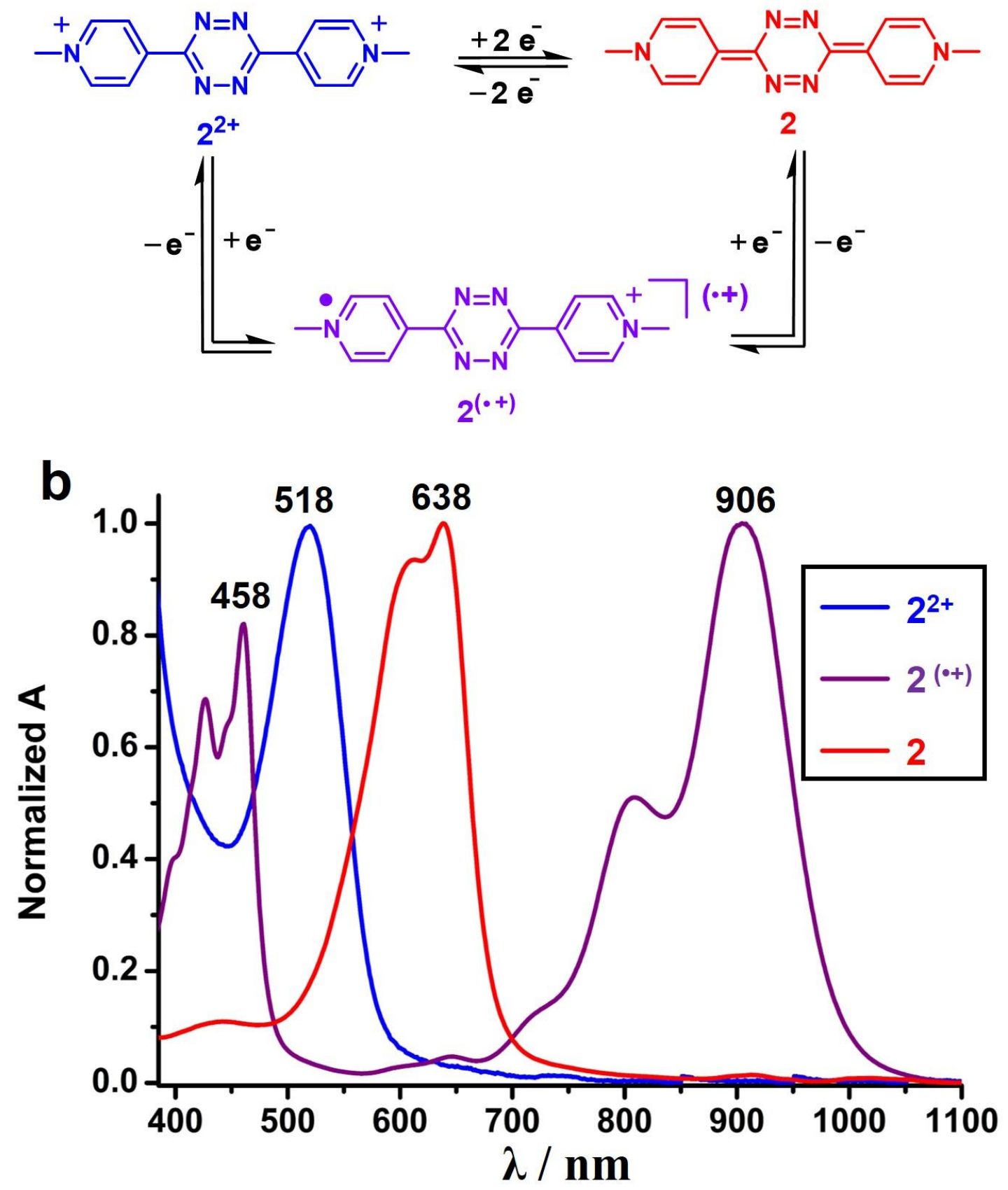

Figure S2. (a) Structural formulas of the three redox states of $2 \cdot 2 \mathrm{PF}_{6}$. (b) Normalized UV-Vis-NIR absorption spectra of $2^{2+/(++) / 0}$, which were obtained by the stepwise addition of $0,1.0$, and 2.0 equiv of $\mathrm{CoCp}_{2}$. All spectra were recorded in Ar-purged DMF solutions at $298 \mathrm{~K}$. 

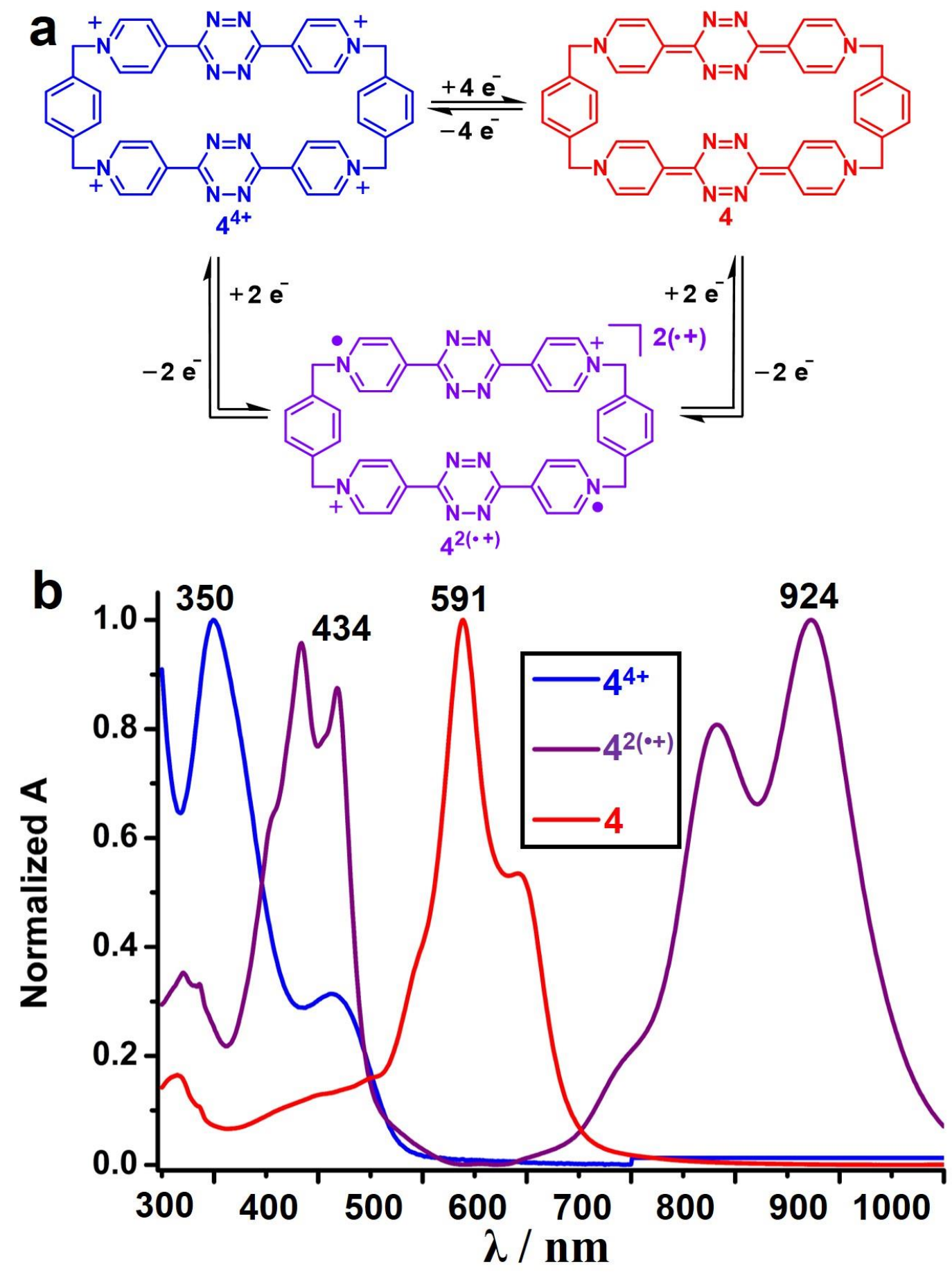

Figure S3. (a) Structural formulas of the three redox states of $4 \cdot 4 \mathrm{PF}_{6}$. (b) Normalized UV-Vis-NIR absorption spectra of $4^{4+/ 2(\cdot+) / 0}$, which were obtained by the stepwise addition of $0,2.0$, and 4.0 equiv of $\mathrm{CoCp}_{2}$. All spectra were recorded in Ar-purged DMF solutions at $298 \mathrm{~K}$. 


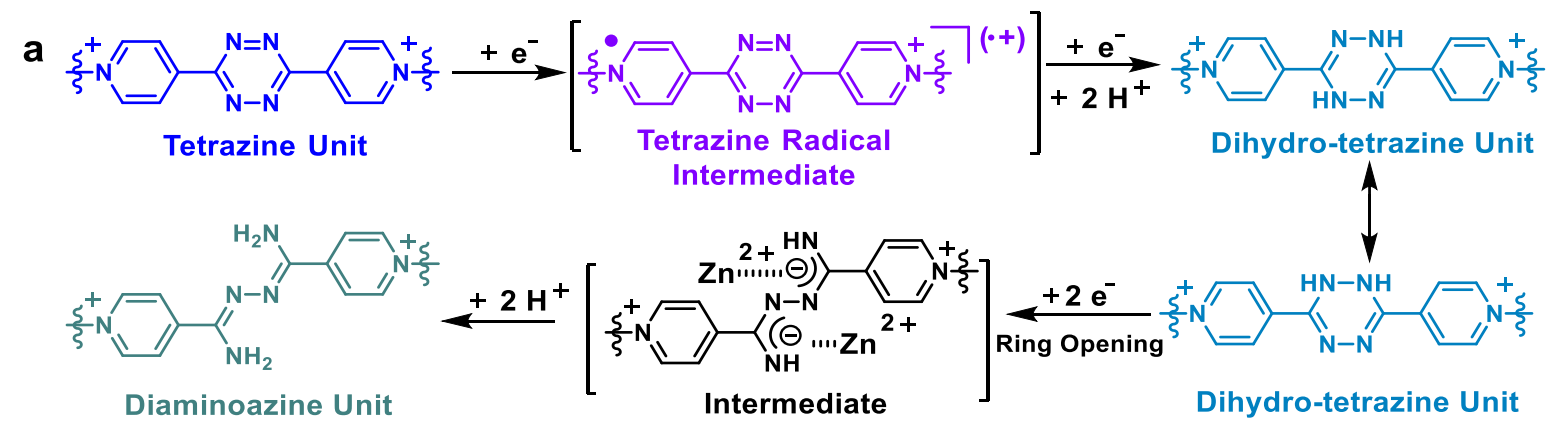

b
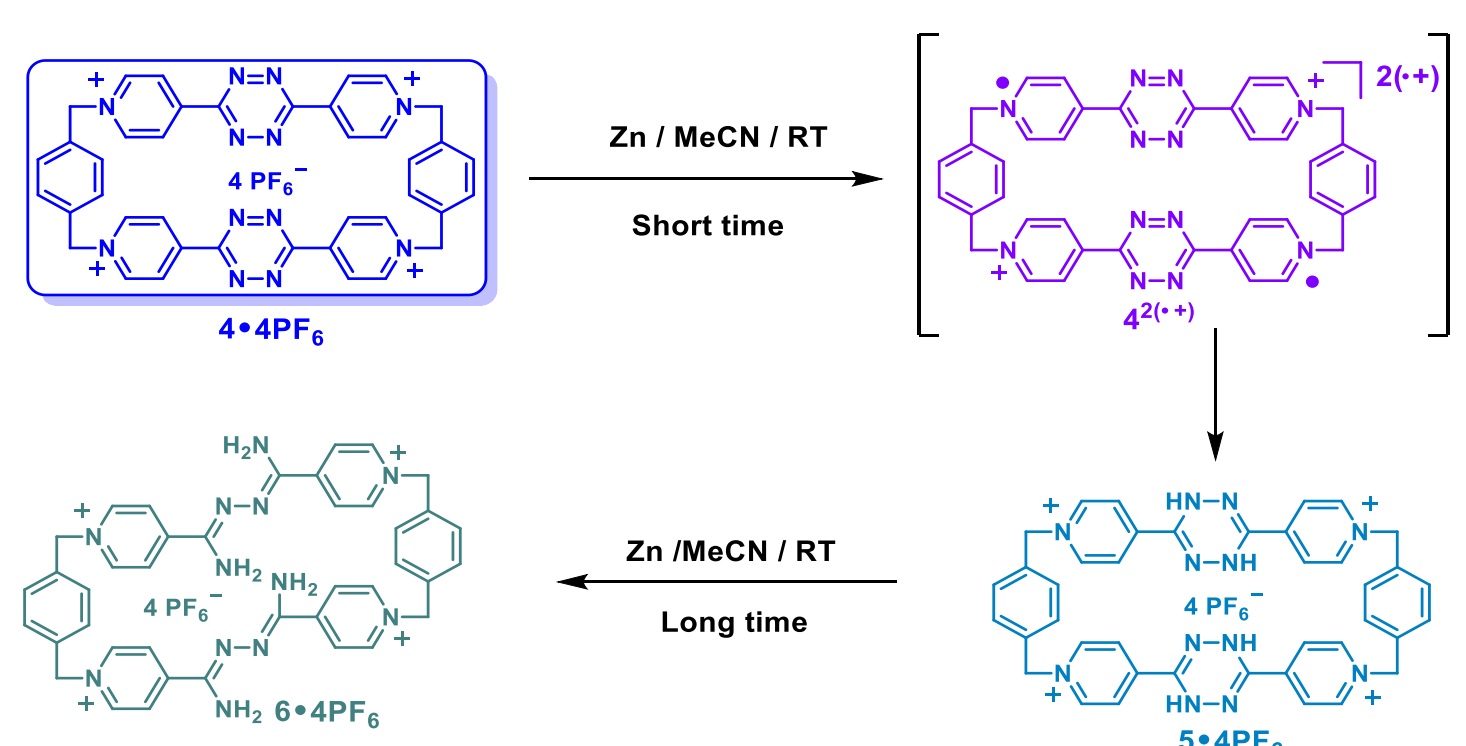

C
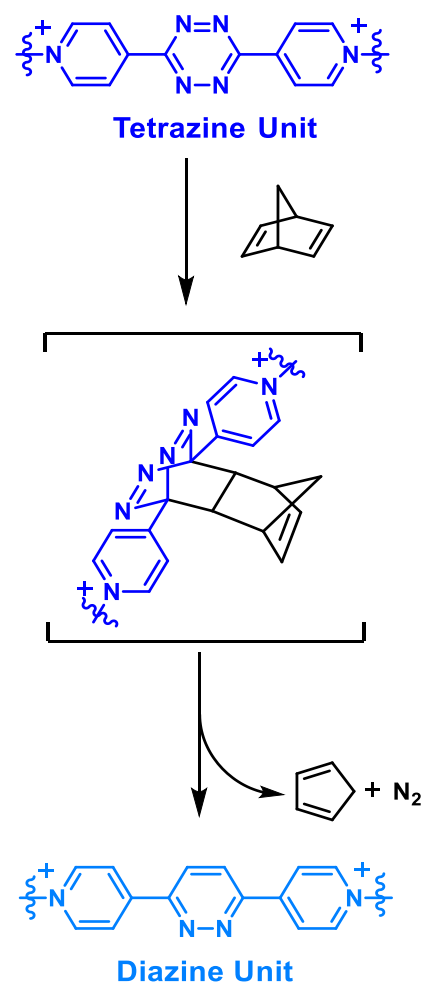

Inverse Electron-Demand Diels-Alder (IEDDA) Reaction

Scheme S7. (a) The mechanism for the metal-mediated reduction, in which radical intermediates are involved. (b) Box-to-box transformation. (c) inverse electron-demand Diels-Alder (IEDDA) reaction. 
Table S1. The Heights, Widths and Lengths of TzBox ${ }^{4+}, \mathbf{D z B o x}^{4+}, \mathbf{D H T z B o x}^{4+}$, and DAzBox ${ }^{4+}$.

\begin{tabular}{|c|c|c|c|}
\hline & Height $(\AA)$ & Width $(\AA)$ & Length $(\AA)$ \\
\hline TzBox $^{4+}$ & 4.53 & 7.29 & 14.15 \\
\hline DzBox $^{4+}$ & 4.33 & 6.99 & 14.49 \\
\hline DHTzBox $^{4+}$ & 4.03 & 9.31 & 13.77 \\
\hline DAzBox $^{4+}$ & & & \\
\hline & 5.69 & & \\
\hline
\end{tabular}


Section E. Regulatable Binding Affinities
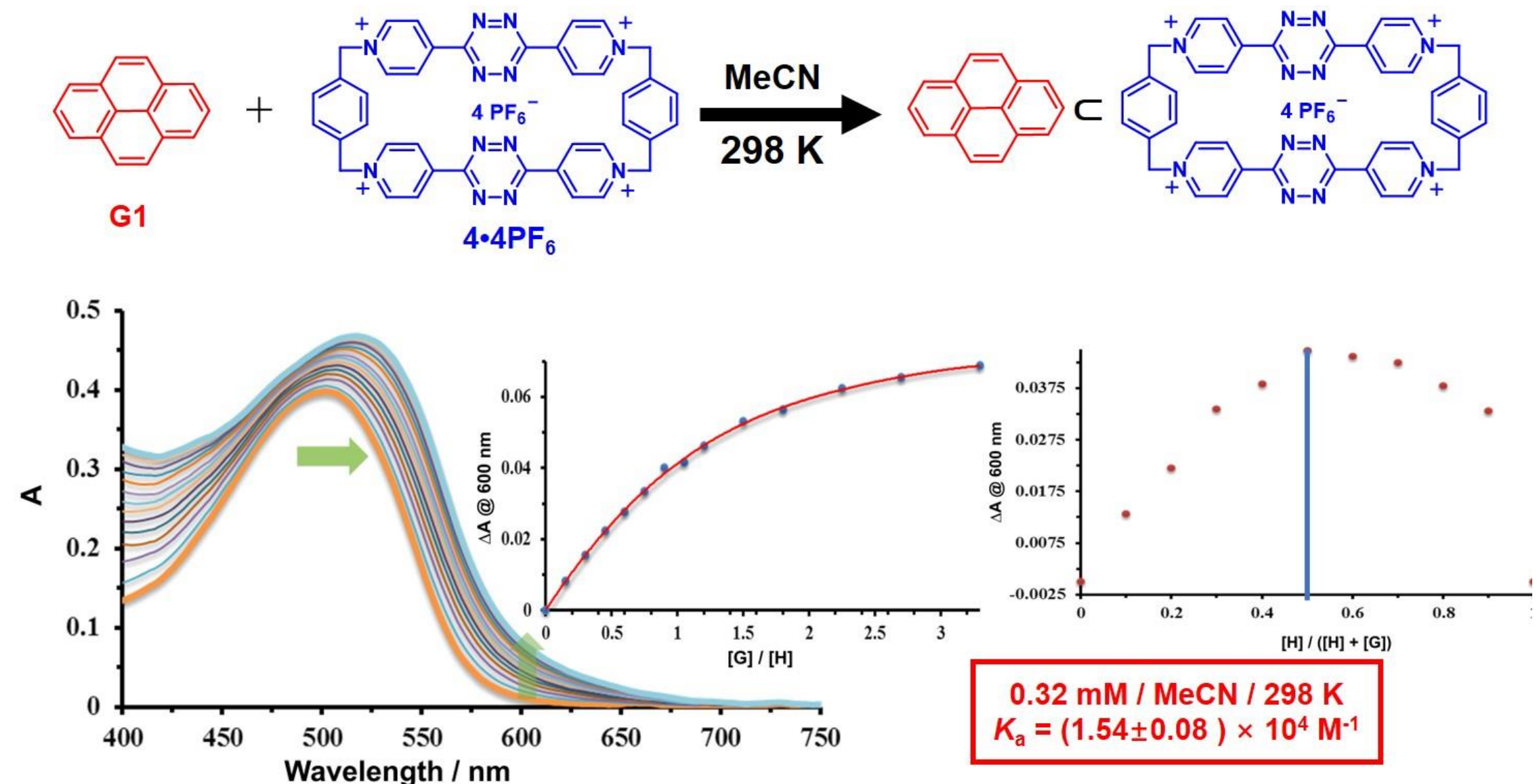

Figure S4. UV-Vis absorption spectrum of $4 \cdot 4 \mathrm{PF}_{6}$ titrated by pyrene in MeCN solution at room temperature. A charge-transfer (CT) band appears. Job plot experiments 1:1 stoichiometry. The binding constant $K_{\mathrm{a}}$ was calculated to be $(1.54 \pm 0.08) \times 10^{4} \mathrm{M}^{-1}$. 

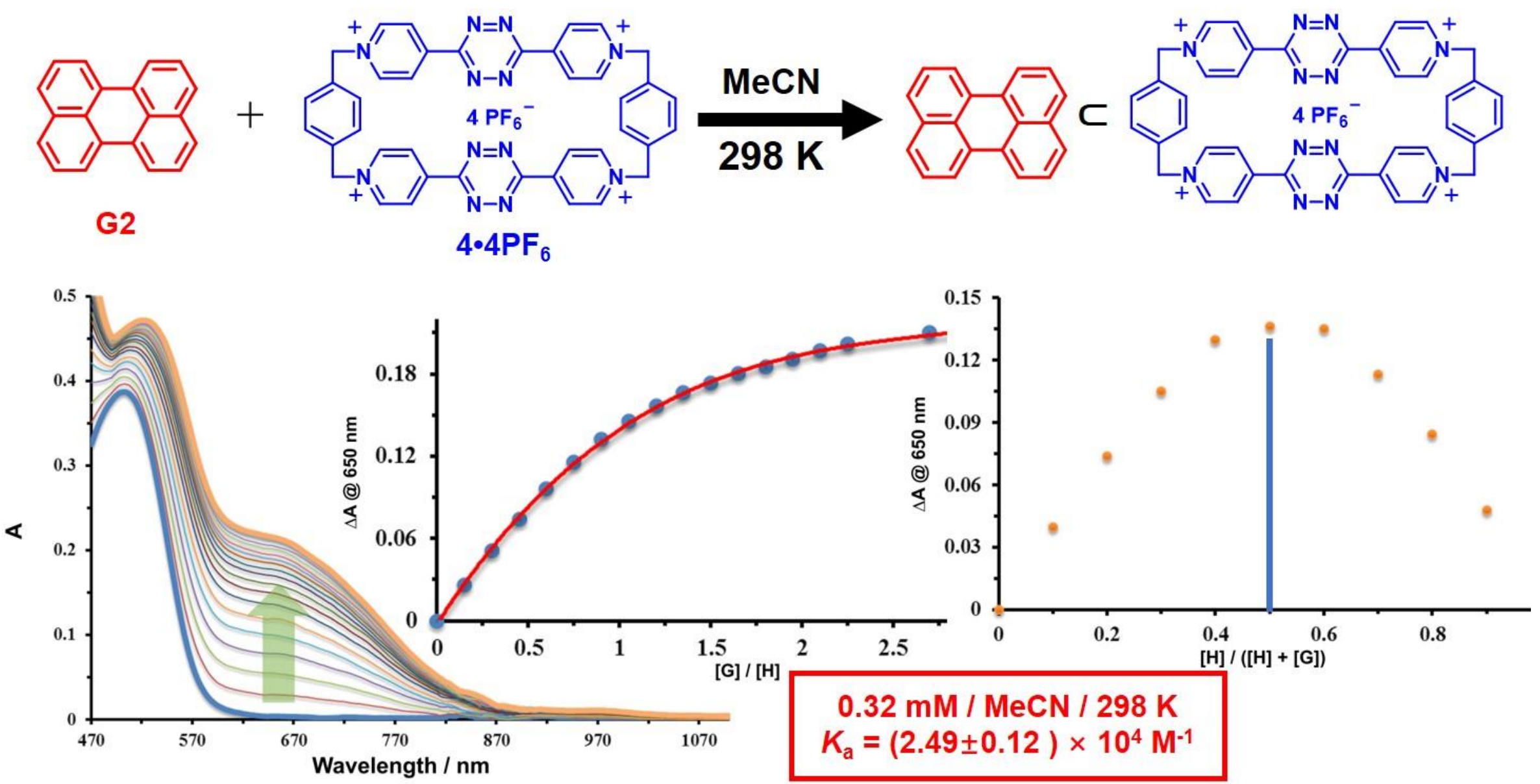

Figure S5. UV-Vis absorption spectrum of $4 \cdot 4 \mathrm{PF}_{6}$ titrated by perylene in $\mathrm{MeCN}$ solution at room temperature. A charge-transfer $(\mathrm{CT})$ band at around $650 \mathrm{~nm}$ appears. Job plot experiments $1: 1$ stoichiometry. The binding constant $K_{\mathrm{a}}$ was calculated to be $(2.49 \pm 0.12) \times 10^{4} \mathrm{M}^{-1}$. 

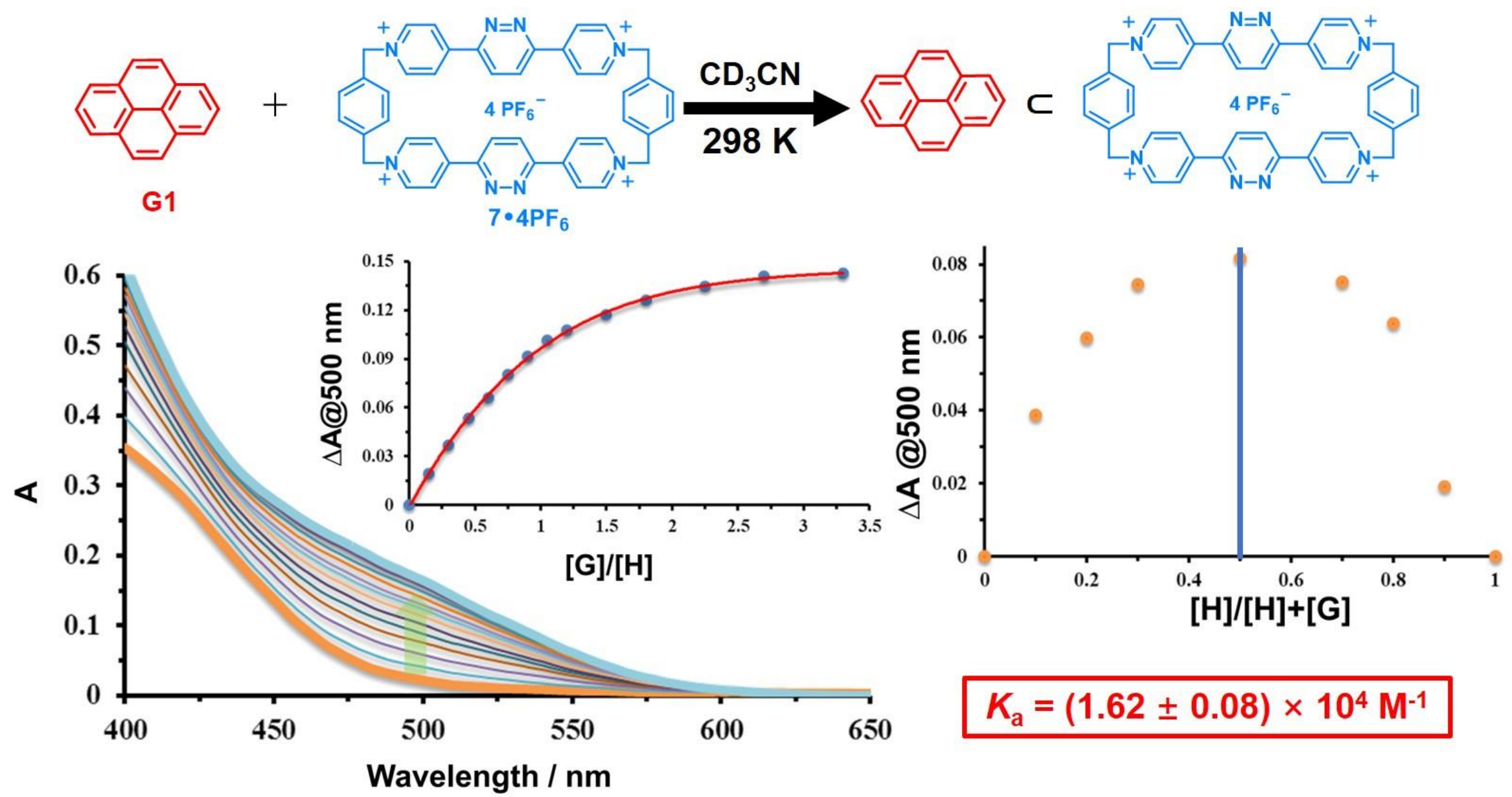

Figure S6. UV-Vis absorption spectrum of $4 \cdot 4 \mathrm{PF}_{6}$ titrated by pyrene in $\mathrm{MeCN}$ solution at room temperature. A charge-transfer $(\mathrm{CT})$ band at around $500 \mathrm{~nm}$ appears. Job plot experiments $1: 1$ stoichiometry. The binding constant $K_{\mathrm{a}}$ was calculated to be $(1.62 \pm 0.08) \times 10^{4} \mathrm{M}^{-1}$. 

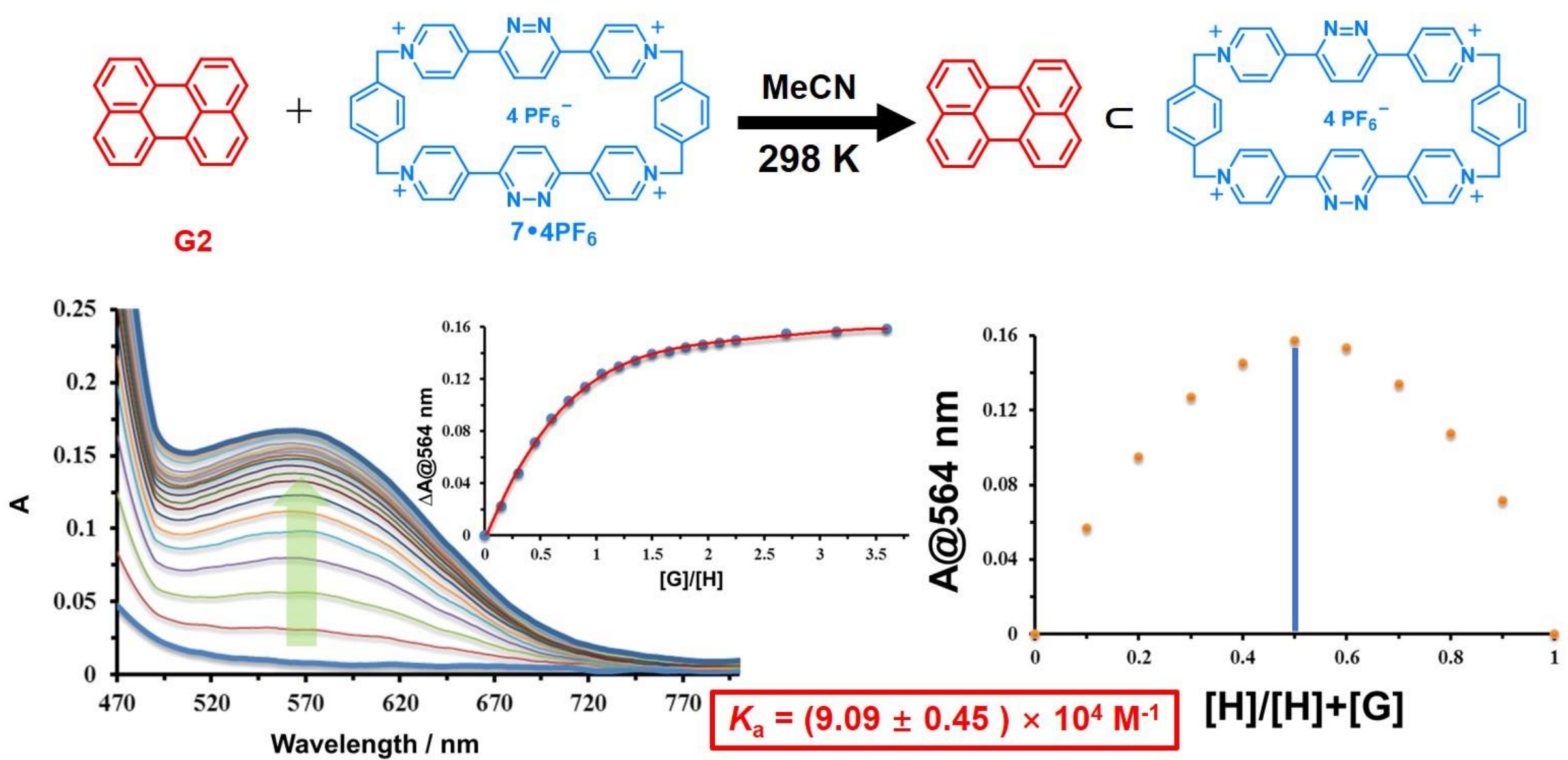

Figure S7. UV-Vis absorption spectrum of $7 \cdot 4 \mathrm{PF}_{6}$ titrated by perylene in $\mathrm{MeCN}$ solution at room temperature. A charge-transfer $(\mathrm{CT})$ band at around $564 \mathrm{~nm}$ appears. Job plot experiments $1: 1$ stoichiometry. The binding constant $K_{\mathrm{a}}$ was calculated to be $(9.09 \pm 0.45) \times 10^{4} \mathrm{M}^{-1}$. 

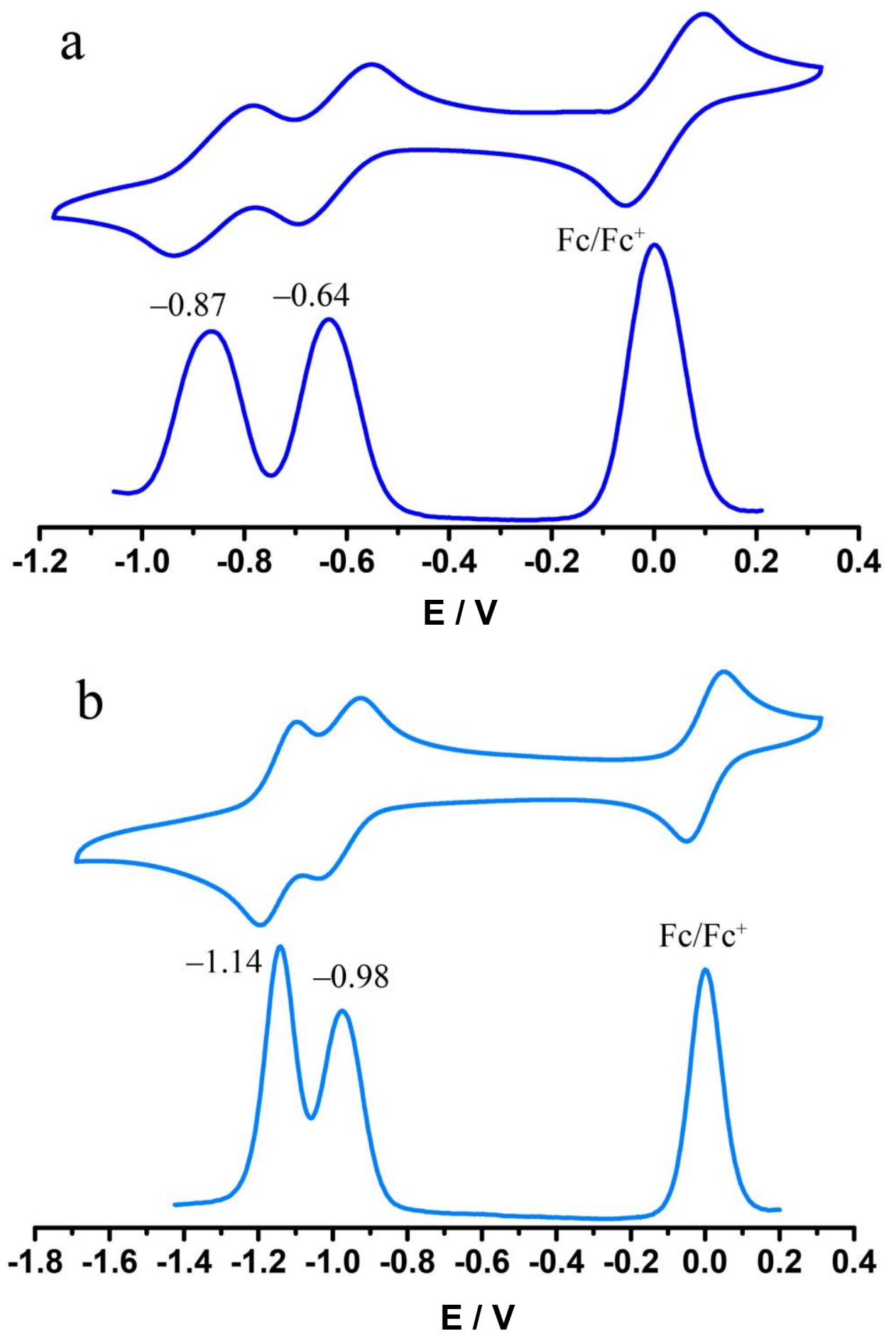

Figure S8. Cyclic voltammogram $(\mathrm{CV})$ and differential pulse voltammetry (DPV) of (a) $\mathbf{T z B o x} \bullet 4 \mathrm{PF}_{6}$ and (b) $\mathbf{D z B o x} \bullet 4 \mathrm{PF}_{6}$ with their reduction potentials highlighted. 

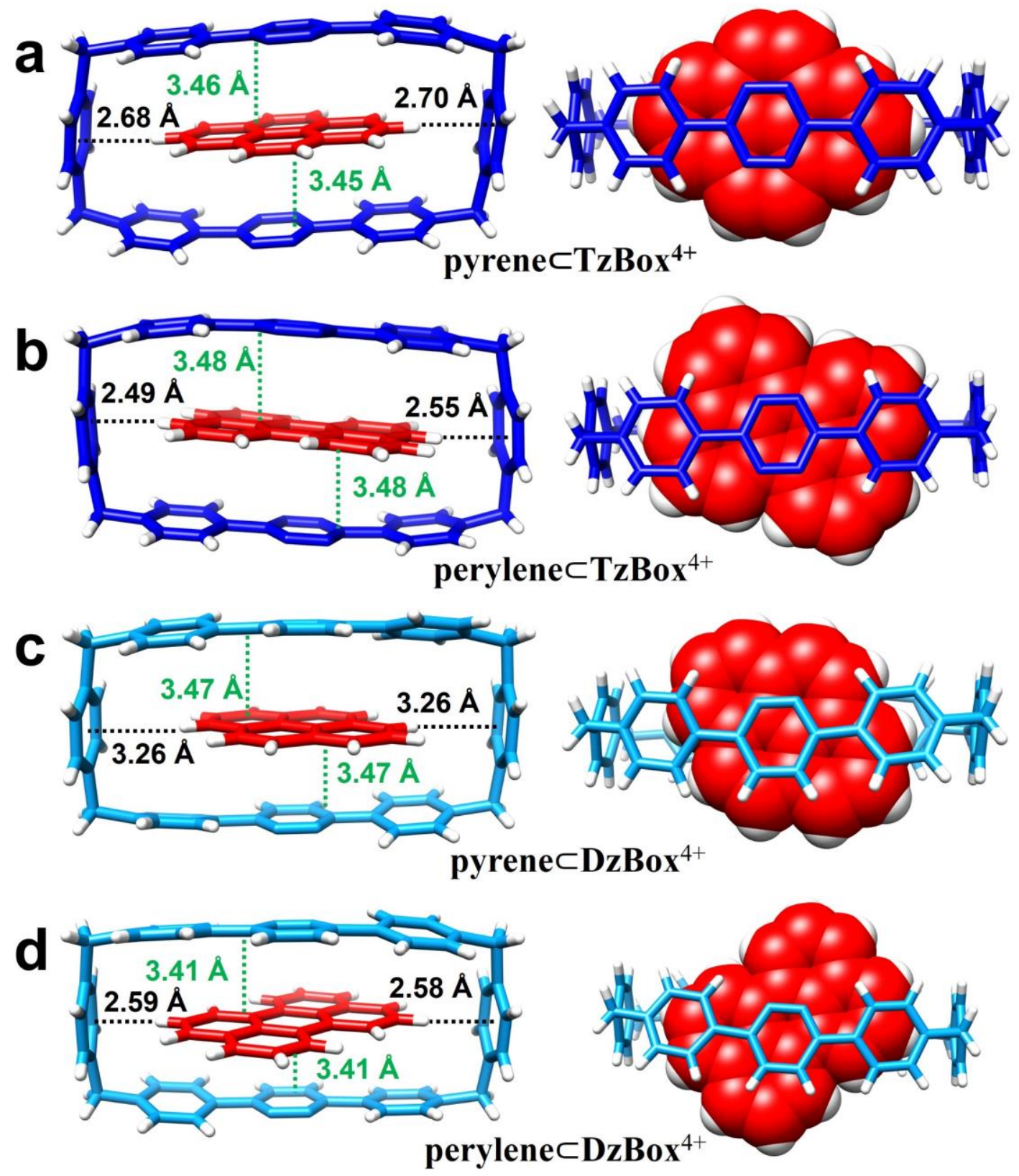

Figure S9. Tubular and mixed tubular/space-filling representations single crystal superstructures of the $1: 1$ inclusion complexes formed by $[\pi \cdots \pi]$ interactions and $[\mathrm{C}-$ $\mathrm{H} \cdots \pi]$ interactions. (a) The pyrene $\subset \mathbf{T z B o x}^{4+}$ 1:1 inclusion complex. (b) The perylene $\subset$ TzBox ${ }^{4+}$ 1:1 inclusion complex. (c) The pyrene $\subset \operatorname{DzBox}^{4+}$ 1:1 inclusion complex. (d) The perylene $\subset$ DzBox ${ }^{4+} 1: 1$ inclusion complex. 


\section{Section F. NMR Spectroscopy}
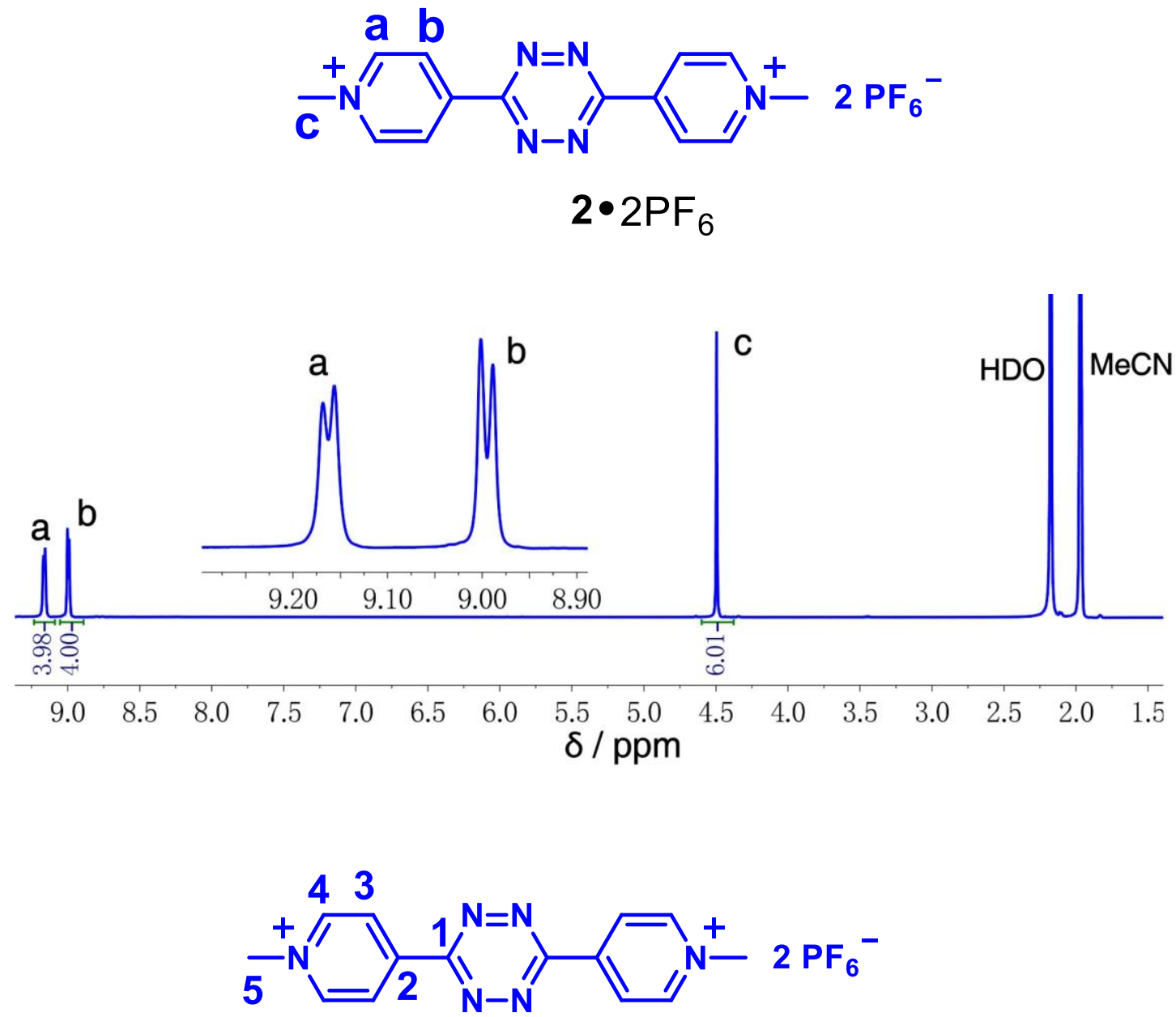

$2 \cdot 2 \mathrm{PF}_{6}$

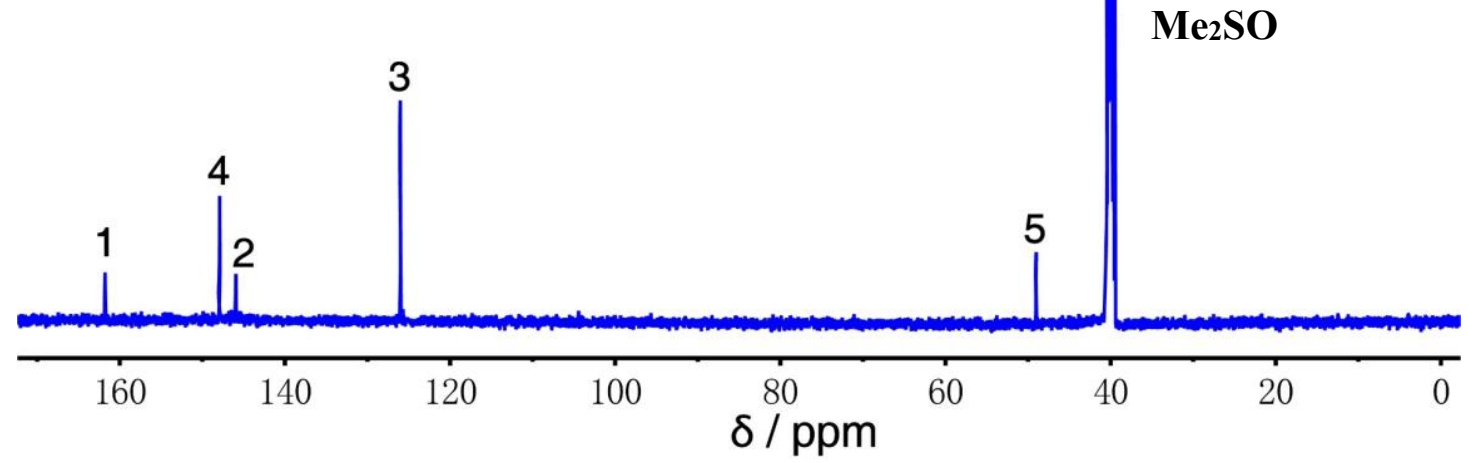

Figure S10. ${ }^{1} \mathrm{H}$ (top) and ${ }^{13} \mathrm{C}$ (bottom) NMR spectra of $2 \cdot 2 \mathrm{PF}_{6}$. 

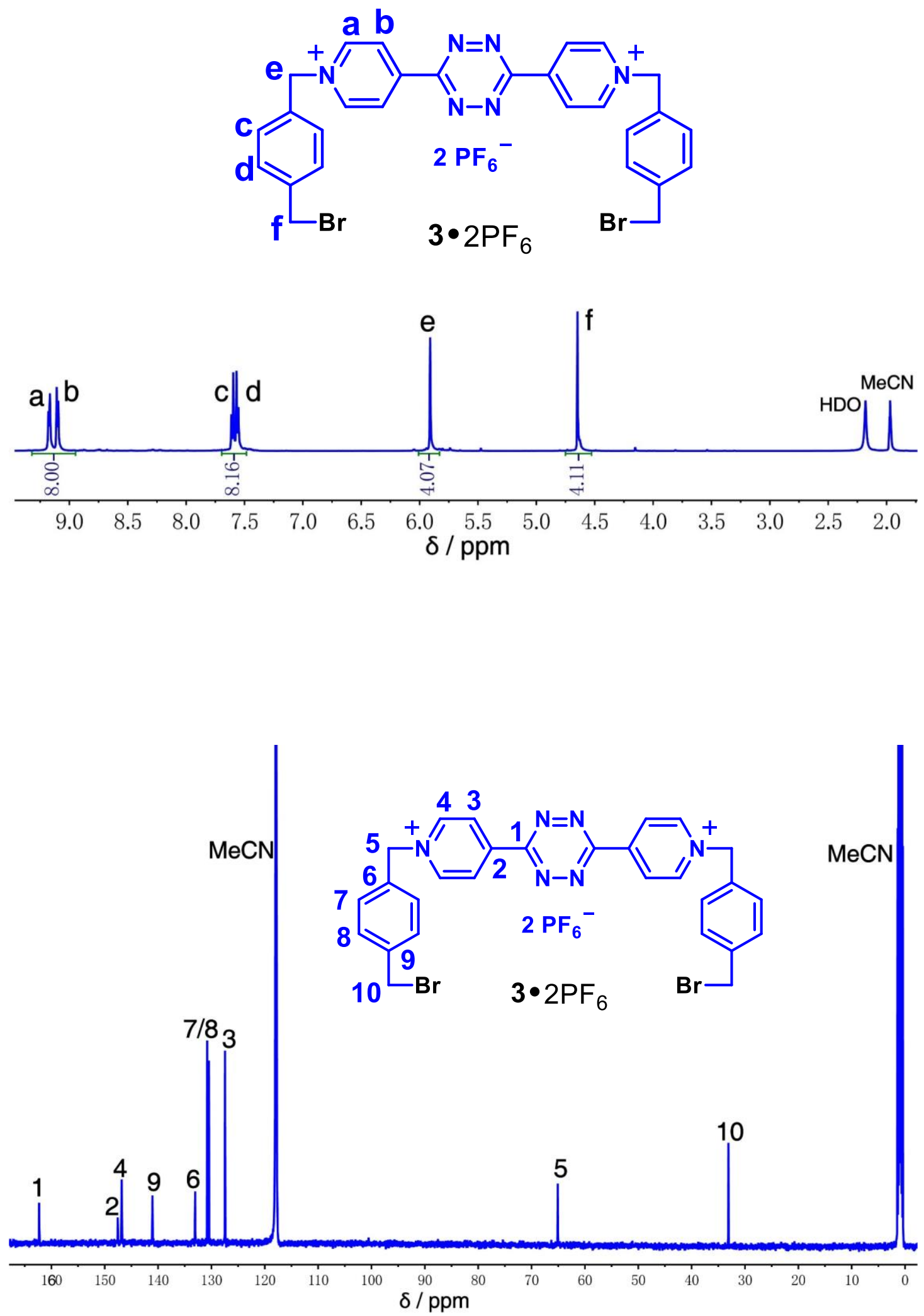

Figure S11. ${ }^{1} \mathrm{H}$ (top) and ${ }^{13} \mathrm{C}$ (bottom) NMR spectra of $\mathbf{3} \cdot 2 \mathrm{PF}_{6}$. 

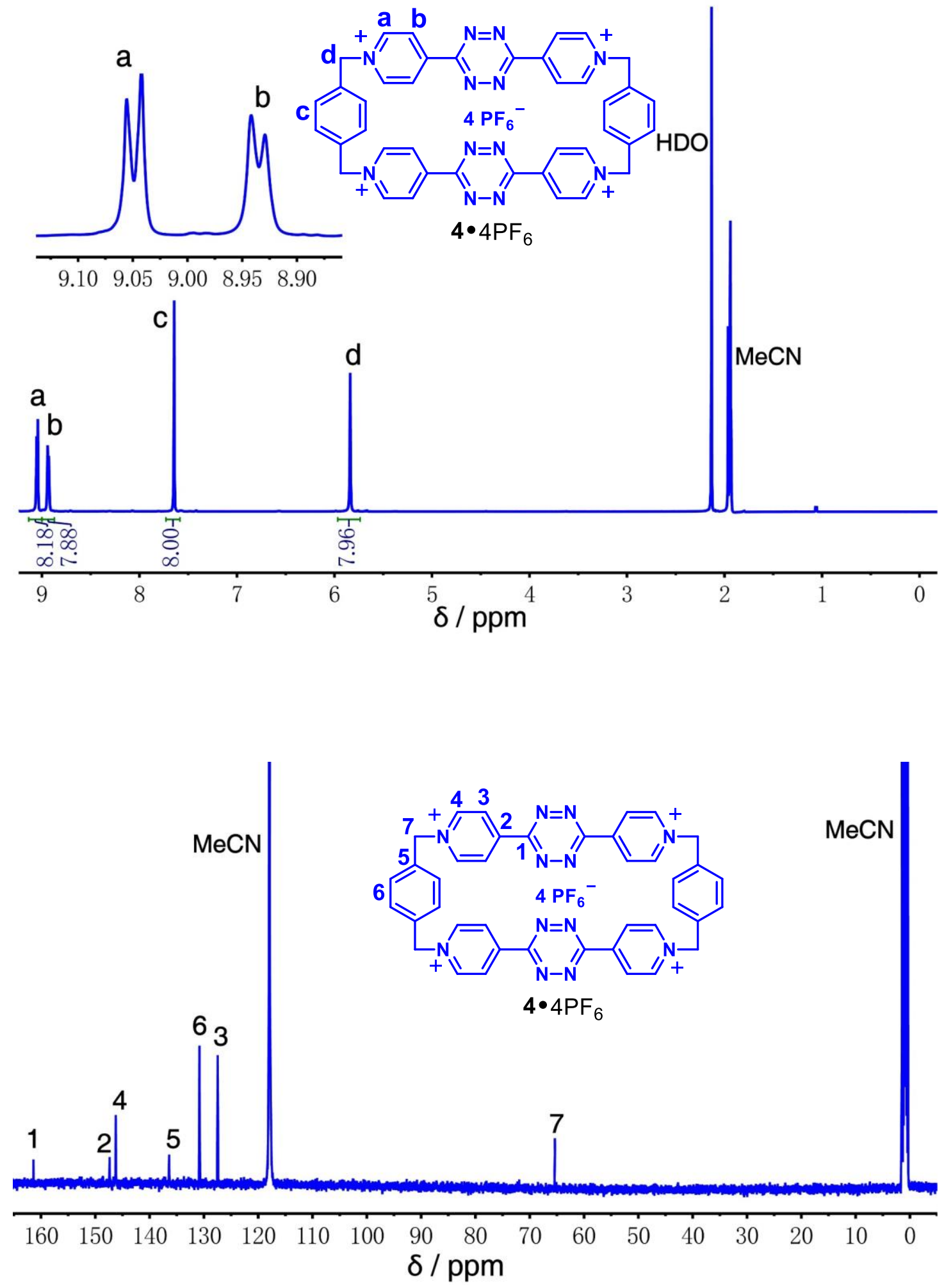

Figure S12. ${ }^{1} \mathrm{H}$ (top) and ${ }^{13} \mathrm{C}$ (bottom) NMR spectra of $4 \cdot 4 \mathrm{PF}_{6}$. 

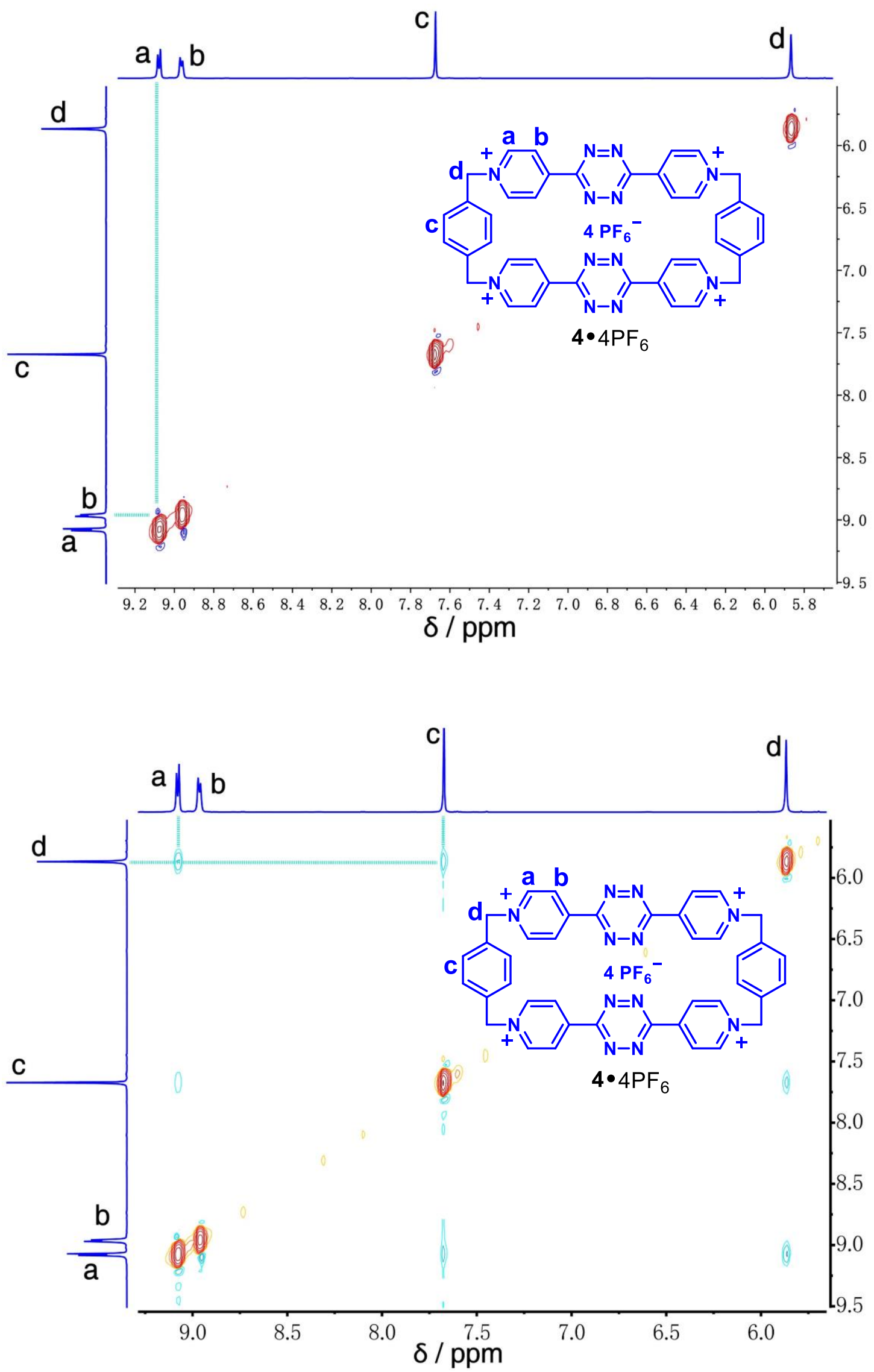

Figure S13. H-H COSY (top) and NOSY (bottom) NMR spectra of $4 \bullet 4 \mathrm{PF}_{6}$. 

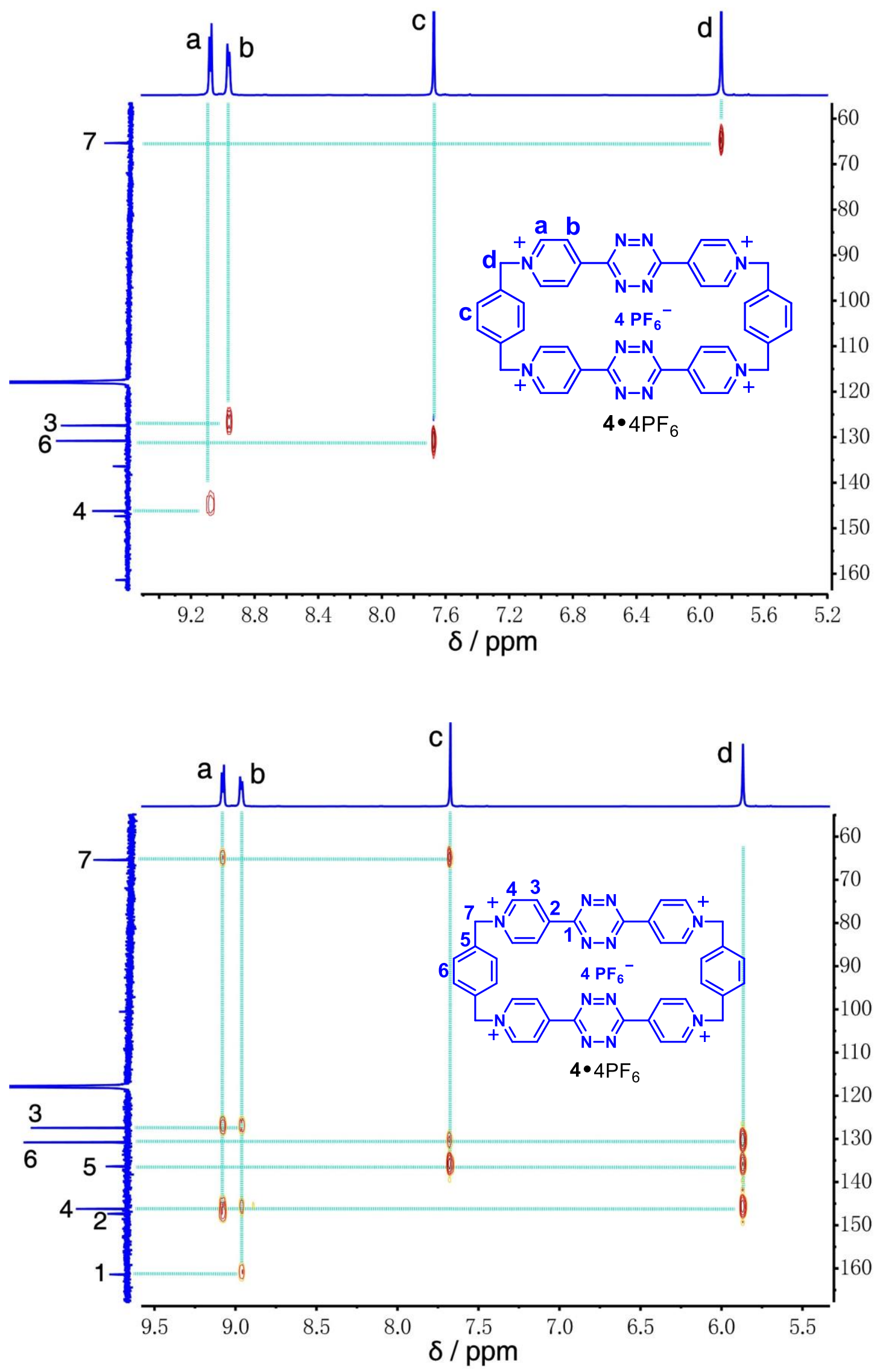

Figure S14. HSQC (top) and HMBC (bottom) NMR spectra of $4 \bullet 4 \mathrm{PF}_{6}$. 

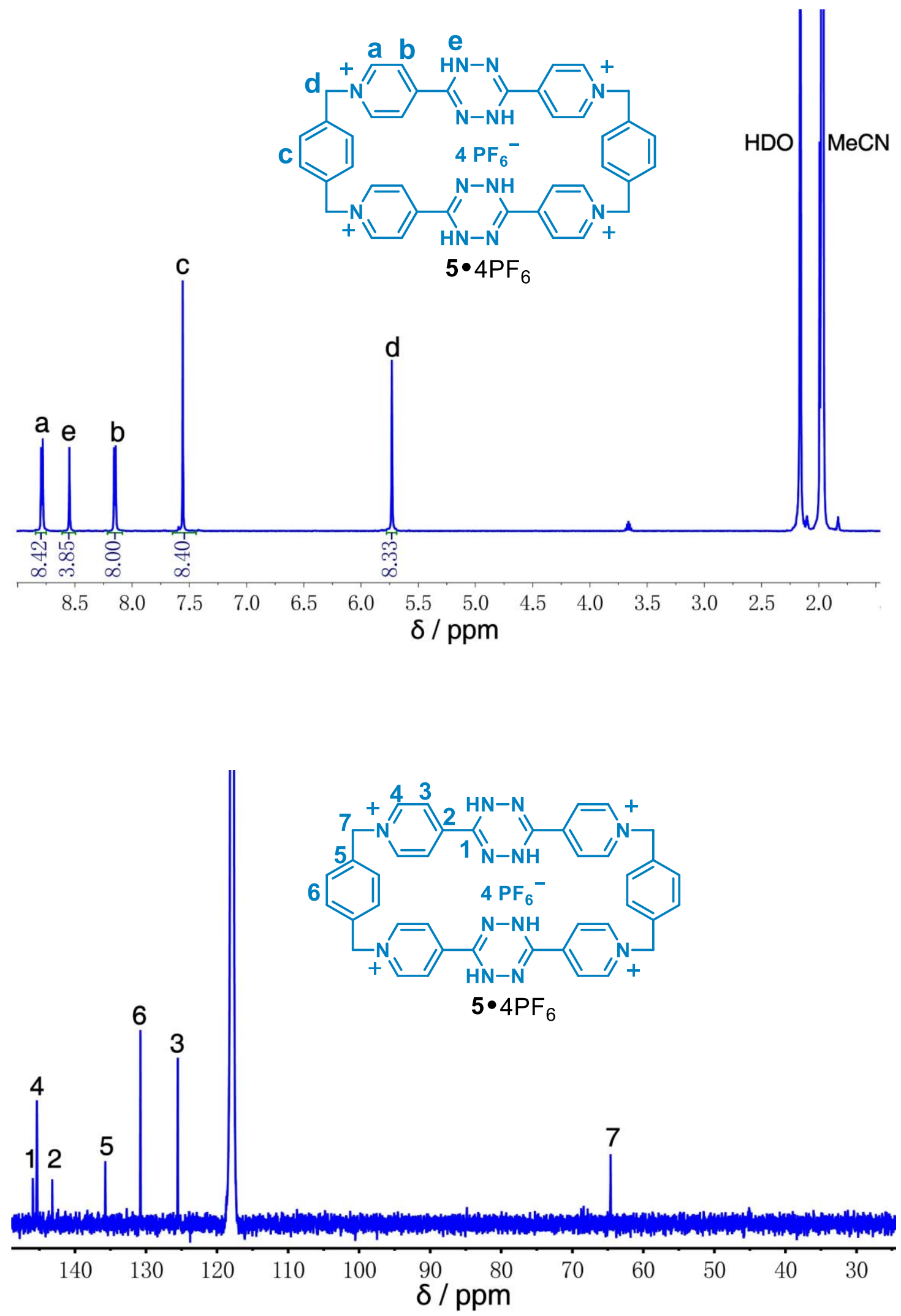

Figure $\mathbf{S 1 5}{ }^{1} \mathrm{H}$ (top) and ${ }^{13} \mathrm{C}$ (bottom) NMR spectra of $\mathbf{5} \cdot 4 \mathrm{PF}_{6}$. 

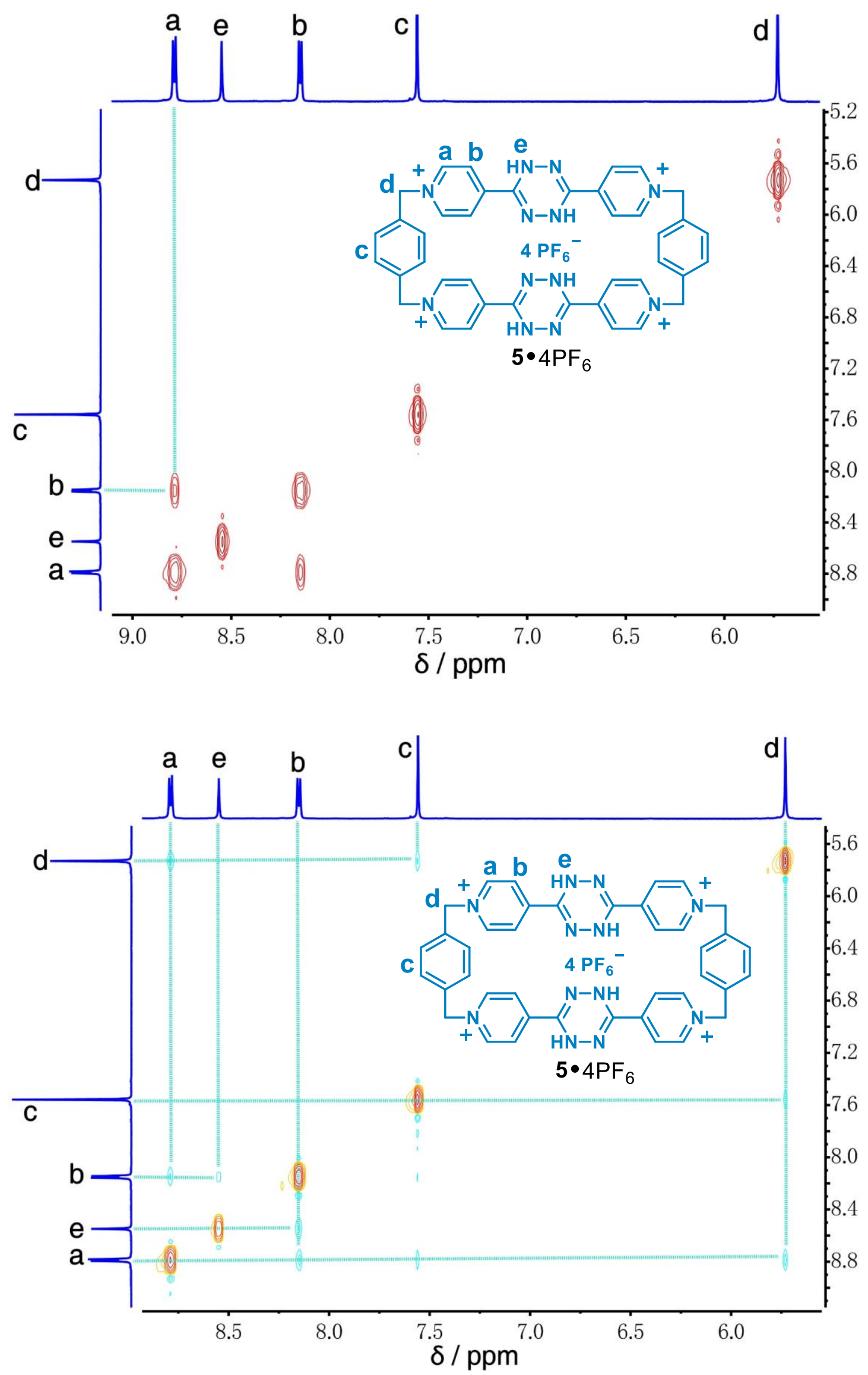

Figure S16. H-H COSY (top) and NOSY (bottom) NMR spectra of 5•4PF 6 . 

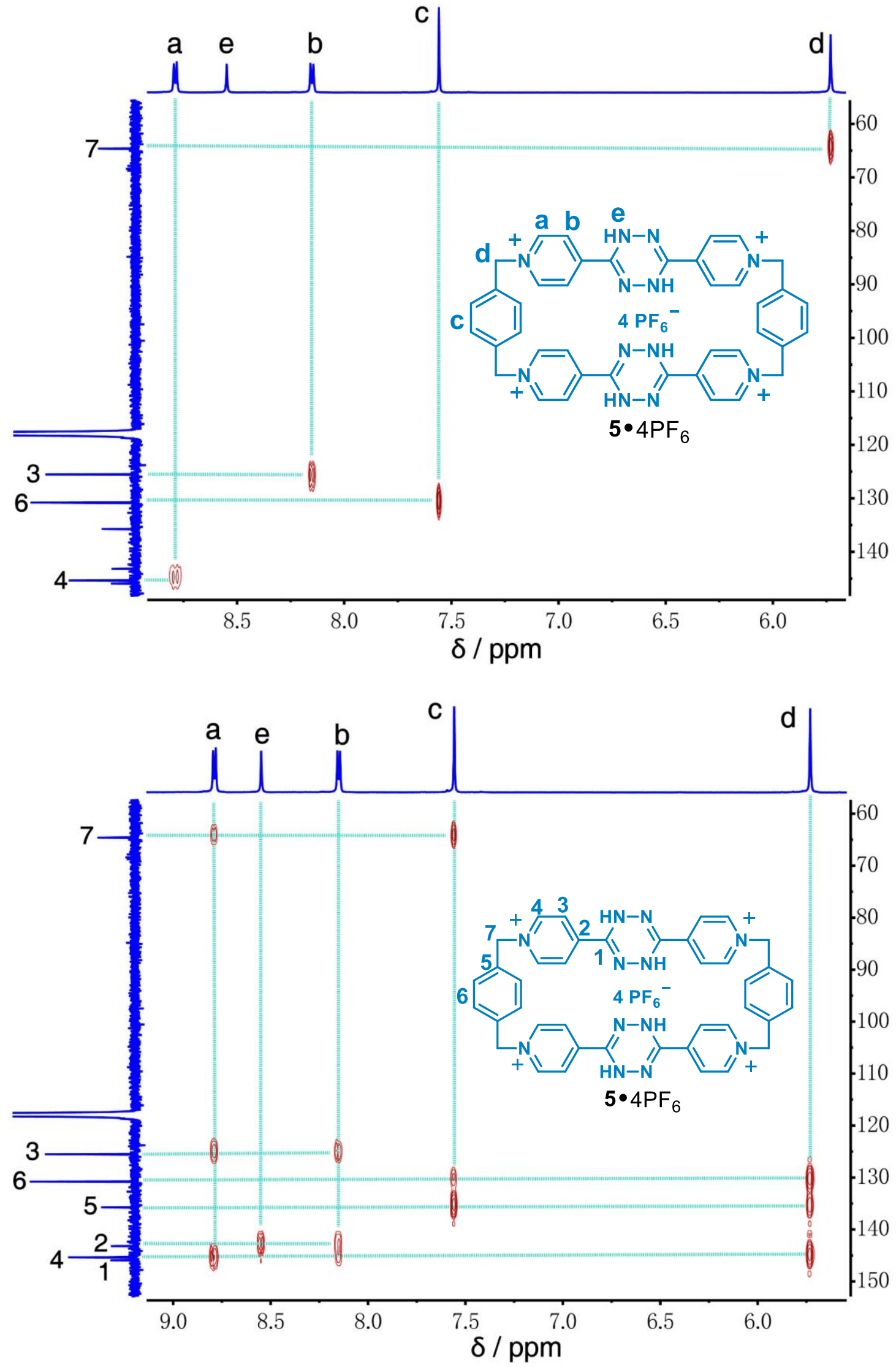

Figure S17. HSQC (top) and HMBC (bottom) NMR spectra of $\mathbf{5} \bullet 4 \mathrm{PF}_{6}$. 

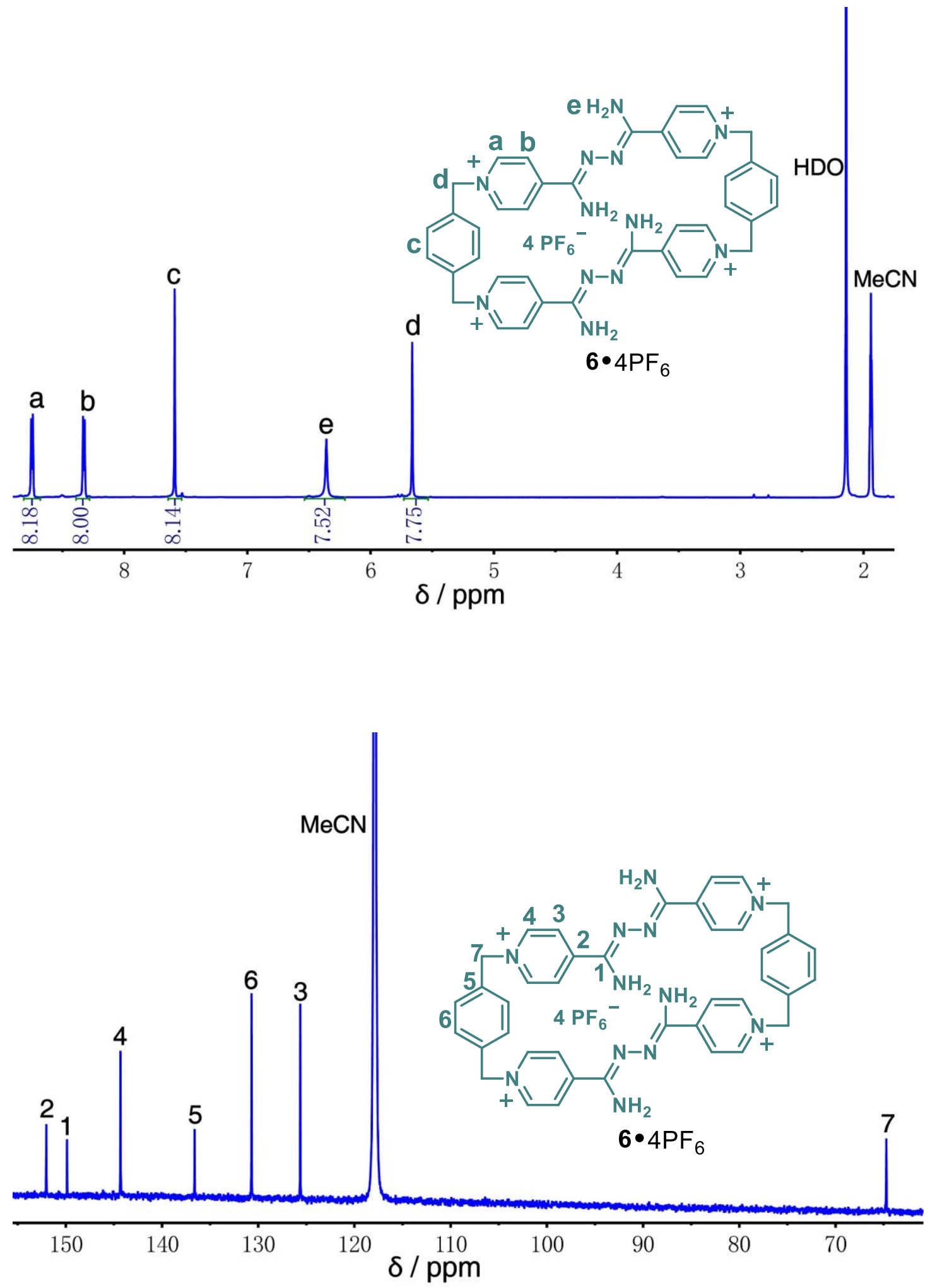

Figure S18. ${ }^{1} \mathrm{H}$ (top) and ${ }^{13} \mathrm{C}$ (bottom) NMR spectra of $6 \cdot 4 \mathrm{PF}_{6}$. 

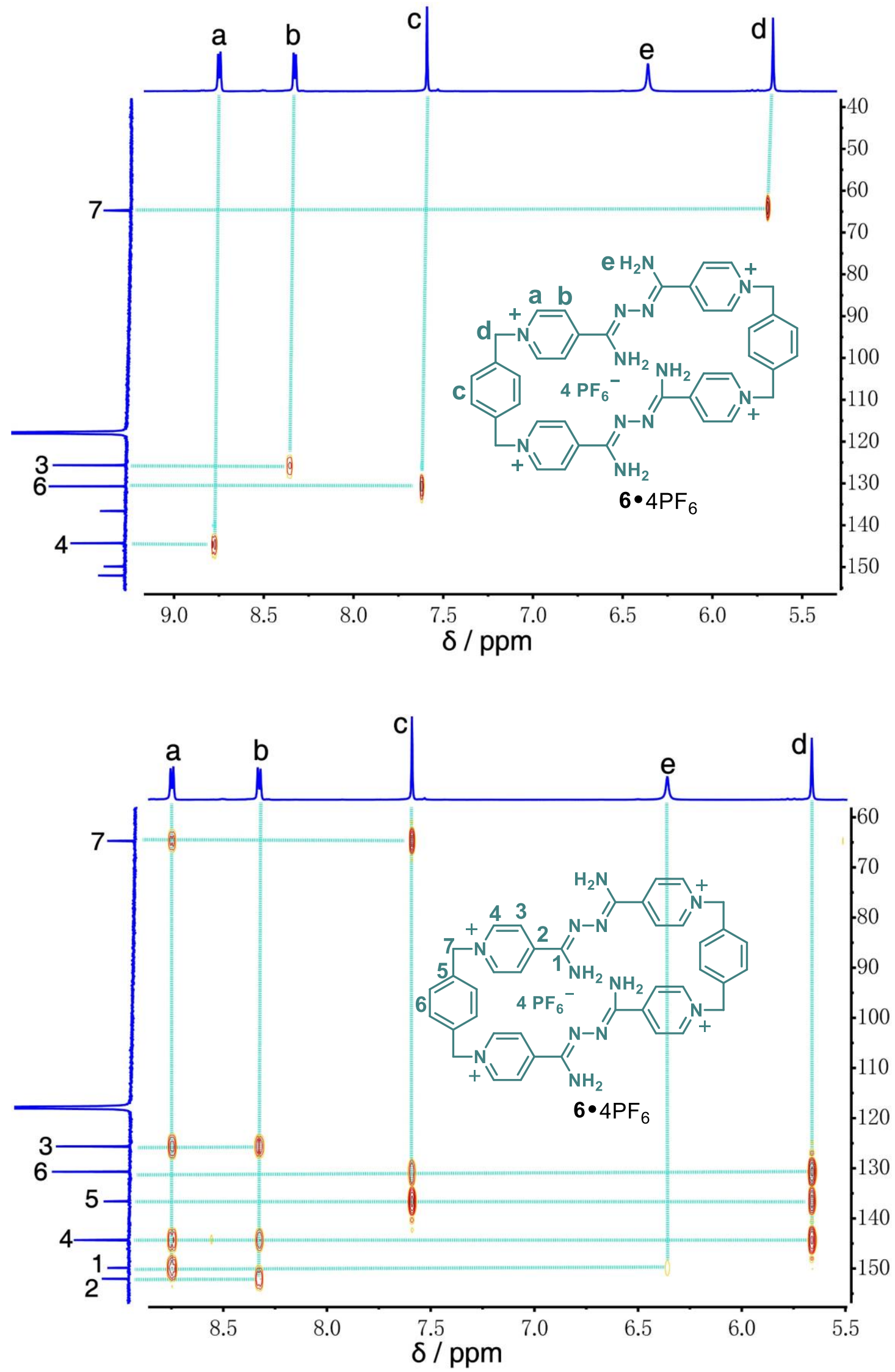

Figure S19. HSQC (top) and HMBC (bottom) NMR spectra of $6 \cdot 4 \mathrm{PF}_{6}$. 

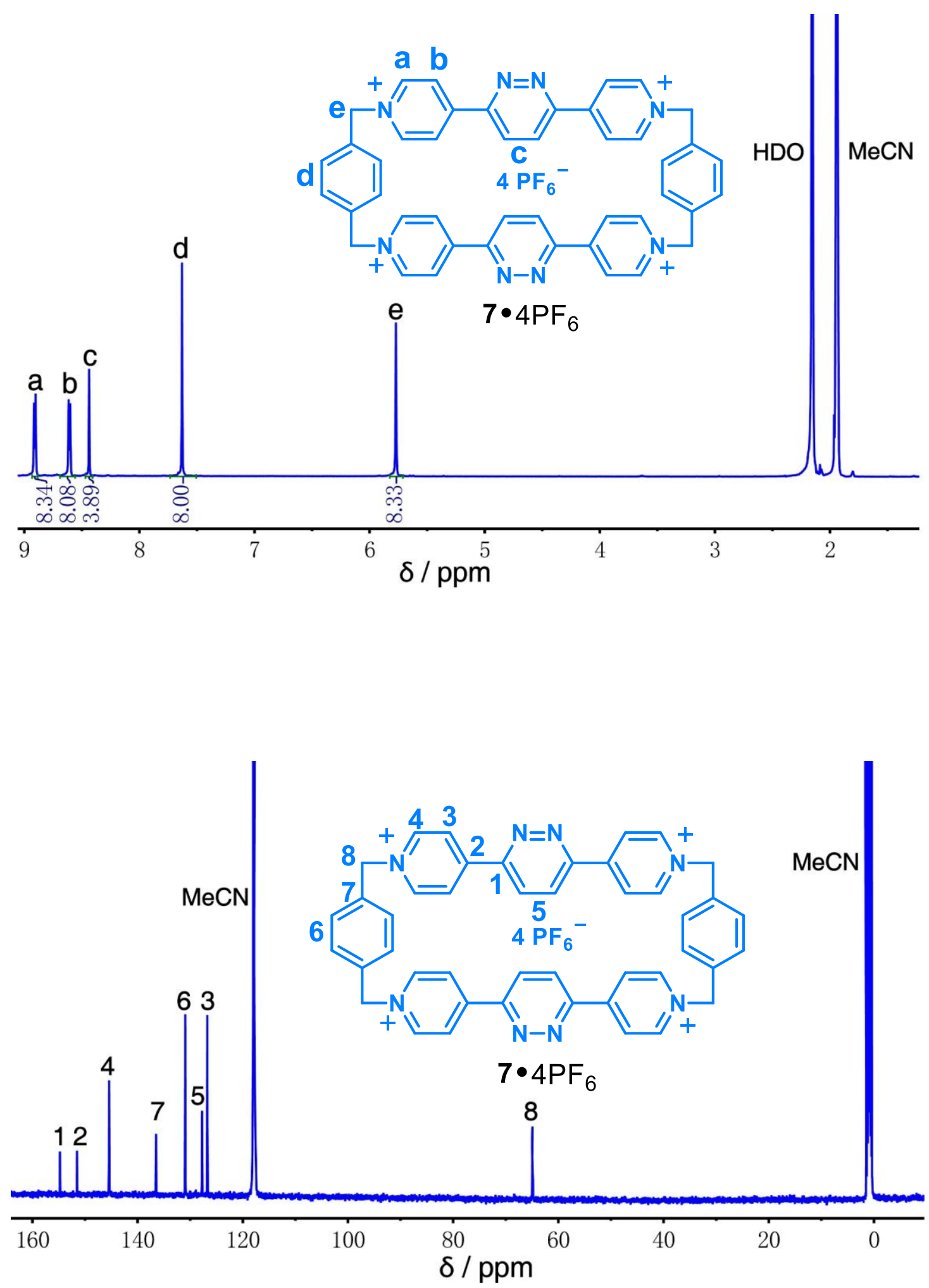

Figure S20. ${ }^{1} \mathrm{H}$ (top) and ${ }^{13} \mathrm{C}$ (bottom) NMR spectra of $7 \bullet 4 \mathrm{PF}_{6}$. 

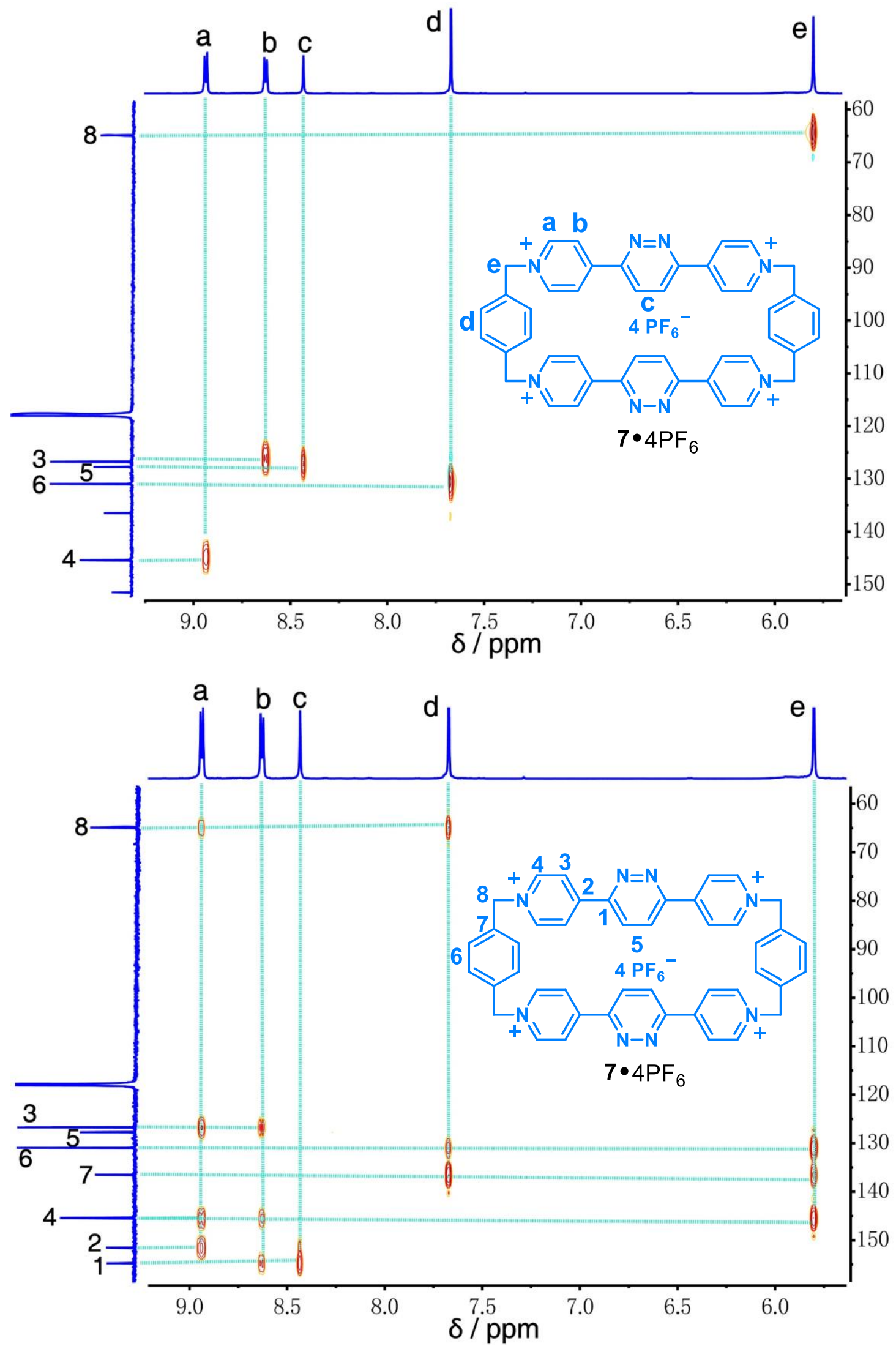

Figure S21. HSQC (top) and HMBC (bottom) NMR spectra of 7•4PF6. 

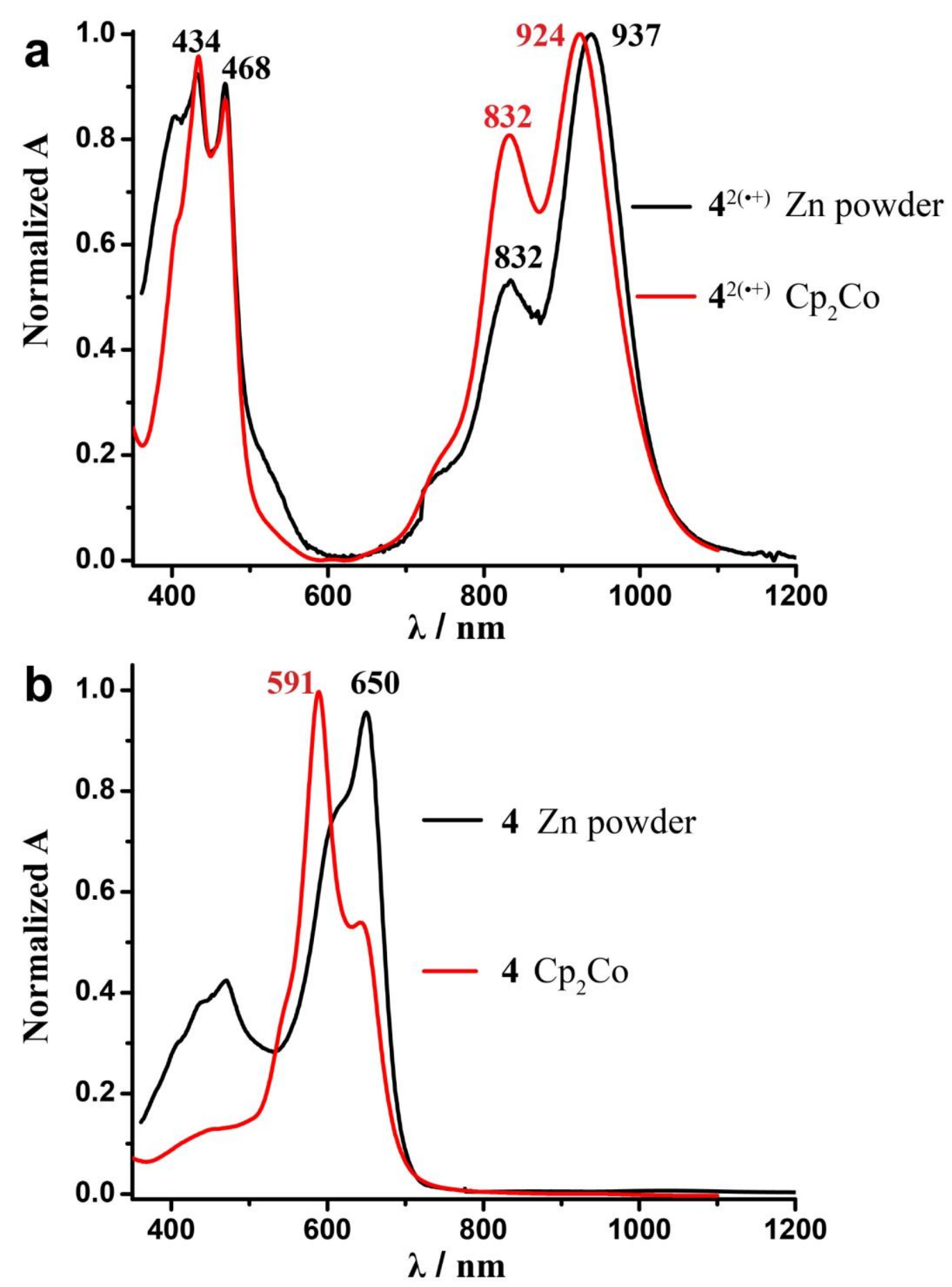

Figure S22. (a) Normalized UV-Vis-NIR absorption spectra of $\mathbf{4}^{2(\cdot)}$, which were obtained by addition of excess amounts (> 20 equiv) of $\mathrm{Zn}$ powder in an Ar-purged dry MeCN solution (black line) and 2.0 equiv of $\mathrm{CoCp}_{2}$ in an Ar-purged dry DMF solution (red line). (b) Normalized UV-Vis-NIR absorption spectra of $\mathbf{4}$, which were obtained by addition of excess amounts (> 20 equiv) of $\mathrm{Zn}$ powder (black line) and 4.0 equiv of $\mathrm{CoCp}_{2}$ in Ar-purged DMF solutions (red line). 


\section{Section G. References}

S1. Y.-C. He et al., Luminescent Anionic Metal-Organic Framework with Potential Nitrobenzene Sensing. Cryst. Growth Des. 2014, 14, 3174-3178.

S2. O. V. Dolomanov, L. J. Bourhis, R. J. Gildea, J. A. Howard, H. Puschmann, OLEX2: A Complete Structure Solution, Refinement and Analysis Program. J. Appl. Cryst. 2009, 42, 339-341.

S3. G. Sheldrick, SHELXT-Integrated Space-Group and Crystal-Structure Determination. Acta Cryst. 2015, 71, 3-8.

S4. G. Sheldrick, A Short History of SHELX. Acta Cryst. 2008, 64, 112-122. 